\title{
The Theory of Industrial Society and Cultural Schemata: Does the "Cultural Myth of Stigma" Underlie the WHO Schizophrenia Paradox? ${ }^{1}$
}

\author{
Bernice A. Pescosolido and \\ Jack K. Martin \\ Indiana University \\ Sigrun Olafsdottir \\ Boston University \\ J. Scott Long \\ Indiana University
}

\author{
Karen Kafadar \\ University of Virginia
}

Tait R. Medina

University of Illinois, Chicago

The WHO's International Studies of Schizophrenia conclude that schizophrenia may have a more benign course in "developing" societies than in the West. The authors focus on this finding's most common corollary: cultural schemata are shaped by the transition from agrarian to industrial society. Developing societies are viewed as traditional, gemeinschaft cultures lacking the stigmatizing beliefs about persons with mental illness held in modern, gesellschaft cultures of developed societies. The Stigma in Global Context-Mental Health Study formalized the cultural myth of public stigma (CMPS) with propositions linking level of development to intolerant, exclusionary, and individualistic attitudes. In 17 countries, the authors find no support for the corollary; where support is found, the findings are opposite expectations, with developed societies reporting lower stigma levels. Reconceptualizing of the cultural landscape on more specific dimensions also produces null or contrary findings. This correction to nostalgic myths of cultural context in developing societies thwarts misguided treatment, policy, and stigma-reduction efforts.

\footnotetext{
${ }^{1}$ Funding for the study was provided by the Fogarty International Center, the National Institute of Mental Health, and the Office of Behavioral and Social Science Research of

(C) 2015 by The University of Chicago. All rights reserved. 0002-9602/2015/12103-0003\$10.00
} 
American Journal of Sociology

If I become psychotic, I'd rather be in India than in Switzerland.

(Shekhar Saxena, WHO, 2006; quoted in Hopper 2008)

An advanced culture broadens more and more the social groups to which we belong with our whole personality; but at the same time the individual is made to rely on his own resources to a greater and greater extent and he is deprived of many supports and advantages associated with the tightly-knit, primary group.

(Simmel 1955)

\section{INTRODUCTION}

An important and continuing controversy surrounding mental illness began in a series of World Health Organization (WHO) studies first fielded in the late 1960s. Eventually spanning 30 research sites in 19 countries, these studies used standardized instruments, training, and research procedures; explicit diagnostic criteria; and local community involvement. The International Pilot Study of Schizophrenia, the Ten Country Study, and the Study of the Determinants of Outcomes of Severe Mental Disorders each revealed enormous heterogeneity in the outcomes of schizophrenia. Importantly, they pointed to a paradox. Despite the lack of general wealth and specific medical resources, "recovery" was more likely in "developing" countries than in "developed" ones (see, e.g., Sartorius et al. 1996; Hopper and Wanderling 2000; Kulhara and Chakrabarti 2001). This widely cited conclusion has been heralded more than once as a singularly important finding (Kohn and Williams 1956; Bromet et al. 1976; Lin and Kleinman 1988; Cohen et al. 2008). As the standard of comparative, cultural-based knowledge across the mental health disciplines, this paradox is a mainstay of textbook discussion and is cherished by some advocacy/consumer groups as a fundamental critique of Western psychiatry.

It is a quintessential sociological finding - the outcome of the intense, private experience of mental illness is fundamentally shaped by public cultures. As Evans-Lacko and her colleagues (2012) have recently shown (albeit in a set of developed countries), individuals with serious mental illness

the U.S. National Institutes of Health (grants 5 R01 TW006374, R01MH082871); Indiana University's College of Arts and Sciences; Rannís - the Icelandic Centre for Research: the Icelandic Research Fund; and Ghent University. Jason Beckfield, Kim Hopper, Alexander Janca, and Tom Smith provided comments on various phases of the project. We acknowledge the contributions of Terry F. White; Alex Capshew, Indiana Consortium for Mental Health Services Research (ICMHSR) associate director and SGC-MHS project manager; and Mary Hannah, ICMHSR production and dissemination manager. 
who live in societies with higher levels of public stigma report greater personal experiences of prejudice and discrimination. Debates about the epidemiology leading to this better prognosis hypothesis and what the WHO studies actually concluded have become even more heated in recent years (Jablensky, Sartorius, and Cooper 1994; Isaac, Chand, and Murthy 2007; Jablensky and Sartorius 2008; Karagianis et al. 2009; Kulhara, Shah, and Grover 2009). ${ }^{2}$ As a counterargument, Patel and colleagues (2006) suggest many reasons and some evidence for why outcomes in middle/low income countries may actually be worse - the overall lack of resources, the relatively scarce services for any medical problem, and the continued reliance on long-term custodial care for mental illness (see also Leff 2001a). Yet to date, the finding that individuals diagnosed with schizophrenia in Latin America, Africa, or Asia seem to "do better" than those who live in the United States and Western Europe has been called "durable" and "remarkably robust" (Hopper and Wanderling 2000, p. 836; Hopper 2008), a "textbook fact" or "axiom" (Burns 2009, p. 203), as well as one of the "oftrepeated tenets of schizophrenia epidemiology" (McGrath 2008, p. 247) and "institutionalized canonicity" (Hopper 2008; see also Cohen et al. 2008).

To separate our concerns, we refer to this set of findings and debates as the WHO schizophrenia paradox (or, in brief here, the WHO paradox). Our aim here is not to enter the better prognosis debate directly. There are no new, high-quality data to adjudicate the epidemiology of recovery on that scale, nor are there ever likely to be. In fact, as Gelder, Mayou, and Cowen (2001, p. 363) note, there are "surprisingly few" long-term follow-up studies in schizophrenia. Cohort studies of "first timers" (i.e., those making their first contact with formal treatment systems) are logistically difficult, requiring a lengthy time frame, specialized training for recruitment, and even more for mixed-method follow up in the community (Link, Andrews, and Cullen 1992; Pescosolido, Brooks-Gardner, and Lubell 1998; Lauronen et al. 2007; Hopper 2008). Rather, our focus is on public stigma, one of the purported mechanisms that underlies the WHO paradox, a cultural mechanism that lies outside of psychiatry's expertise and purview.

Both sides of the debate agree that the reasons for the underlying heterogeneity in schizophrenia outcomes are far from clear (see Leff et al. 1992; Kulhara and Chakrabarti 2001; Jablensky 2009; Thirithalli and Jain 2009). Yet, neither this lack of clarity nor absence of data has stopped considerable speculation. In particular, sociocultural factors or the "general environment in which patients live" (Gelder et al. 2001, p. 367) have

\footnotetext{
${ }^{2}$ See contributions to a forum discussion led by the editors of Schizophrenia Bulletin (http://www.schizophreniaforum.org/for/live/detail.asp?liveID=59).
} 
been implicated as the root of difference (Jablensky et al. 1992; Hopper and Wanderling 2000; Patel et al. 2006; Kulhara et al. 2009). Specifically, lower and middle income countries, often described as "collectivist cultures" (e.g., Lee et al. 2005), are seen as emphasizing tolerance (rather than prejudice), inclusiveness (rather than exclusion), and collective (rather than individual) responsibility. In essence, these are core aspects of stigma, or the stereotypes, prejudice, and discrimination that accompany mental illness (Pescosolido and Martin 2015). Thus, it follows that public stigma, the attitudes and beliefs held in the larger cultural context, forms at least part of the constituted general environment. Consequently, the argument is that individuals with schizophrenia in developing countries face, on average, less stigma than their counterparts in developed countries. This corollary of the WHO paradox has wide acceptance. Yet, whether and how the larger social context, particularly level of development, shapes cultural scripts for responses to people with schizophrenia is essentially absent data.

We develop the cultural myth of public stigma (CMPS) as a set of propositions designed to conceptualize the corollary about the connection between societal forms and cultural schemata, those aspects of cognition that guide social response, including interaction (Swidler 2001; Ridgeway 2006). We ask three questions about the public stigma of mental illness on a global scale. First, can the cultural schemata of some countries be characterized empirically as more intolerant, more exclusionary, and more individualistically oriented toward individuals with mental illness than other countries? Second, if so, is public stigma lower in developing countries as suggested by the WHO schizophrenia paradox corollary? Third, is public stigma associated with overarching but more specific cultural contexts of intolerance, exclusion, and individualism that translate into targeted attitudes, beliefs, and behavioral predispositions that devalue persons with mental illness? The Stigma in Global Context-Mental Health Study (SGC-MHS), the first globally targeted, theoretically and methodologically coordinated empirical examination of the public stigma of mental illness, provides nationally representive data uniquely capable of addressing these questions.

Whether these data support these propositions has important sociological, psychiatric, and policy implications. The link between public cultures and personal lives is central to the sociological perspective. Supportive findings also provide a pathway to improving the life chances of individuals with schizophrenia through treatment and discharge planning protocols that draw from traditional cultural schemata. However, if testing hypotheses derived from CMPS speculations on the corollary of the WHO paradox suggests that the "kinder, gentler" cultures of traditional societies are a myth, then cultural stereotypes tied to development must be replaced with alternative theories and examined with other data to inform policy and practice. 


\section{THEORETICAL BACKGROUND: THE THEORY OF INDUSTRIAL SOCIETY, PUBLIC STIGMA, AND SCHIZOPHRENIA}

\section{The WHO Schizophrenia Paradox}

Ironically, the International Studies of Schizophrenia (ISoS) were conceptualized and designed to counter other extant "myths" about the causes and outcomes of schizophrenia that had long held sway in psychiatry (Jablensky 2009). Prominent among these was the contention that, since mental illnesses arise from disease-inducing conditions of modernity, schizophrenia did not exist in premodern societies. Pioneers of psychiatry (e.g., Emile Kraeplin in the late 1800s) also held that the natural outcome of schizophrenia was progressive degeneration and premature death (Feiner and Frese 2009; Jablensky 2009; Peralta and Cuesta 2011). Such beliefs shaped what individuals and families were told in treatment and, on the societal level, propelled the development of long-term, rurally located institutions to counter the stresses of industrialization (Grob 1991; Pescosolido and Rubin 2000). Yet, these "asylums" came to be seen as a major part of the problem, providing little treatment and even less social activity, furthering the degradation of social skills, accompanied by the stigma of being a "mental patient" (Goffman 1961).

Armed with a global understanding of culture-bound syndromes from anthropology (e.g., Waxler 1979) and clinical knowledge of similar symptom sets caused by infectious and parasitic disease (African trypanosomiasis; Jablensky 2009), the International Pilot Study of Schizophrenia (IPSS) charted the global prevalence and course of schizophrenia. The IPSS documented better outcomes (at two and five year points) in India, Columbia, and Nigeria compared to Denmark, the Union of Soviet Socialist Republics, the United Kingdom, and the United States (Sartorius, Jablensky, and Shapiro 1978). However, recruiting from hospital admissions raised concerns of bias stemming from including participants at different points in their illness careers. In response, the Ten-Country Study recruited only "first-episode" cases assessed upon their first contact with community or hospital services (Jablensky 2009). Again, findings supported cross-national outcome heterogeneity along the developing-developed axis. ${ }^{3}$ Finally, the most methodologically rigorous analyses of cross-national outcome data on schizophrenia combined the incidence cohorts of the Determinants of Outcome of Severe Mental Disorders, the Reduction of Disability Studies, and

\footnotetext{
${ }^{3}$ Country was not the only factor associated with better outcomes in five of the six criteria - acute onset, marital/cohabitating status, and access to supportive networks mattered (Sartorius et al. 1986; Jablensky et al. 1992). In fact, the most consistent determinant of negative outcomes was unremitting psychosis in first two years (Feiner and Frese 2009).
} 
two additional samples from Hong Kong and Madras/Chennai. Again, the findings supported the developing/developed country difference in outcomes. Importantly, these studies went further to assess (and eventually rule out) the possible interference of selection bias (i.e., "leakage" in case identification), diagnostic elasticity, and dropout in developing countries (Hopper and Wanderling 2000; Hopper, Harrison, and Wanderling 2007; Hopper 2008).

In sum, while debate about findings continues, the cross-national difference in outcomes along the developing-developed divide appears to be solid although often misquoted. Jablensky and Sartorius (2008, p. 254) provide a nuanced summary of study findings, clarifying that the "outcome of patients in the developing countries was not uniformly better," nor were "the proportions of continuous unremitting illness" different in the two types of countries. Yet, developing societies reported better outcomes on three major counts: (1) complete clinical remission was significantly more likely in developing nations, (2) more individuals had longer periods of community functioning, and (3) fewer needed to be continuously on antipsychotic medication. Such differences in the public, provider, and policy worlds of psychiatry are highly noteworthy.

Purported Mechanisms Underlying the WHO Paradox:

The Level of Development Corollary

No shortage of explanations for the better prognosis hypothesis have been advanced, particularly in scholarly articles and textbooks in the mental health disciplines (i.e., psychiatry, psychology, social work, nursing). Not surprisingly, some suggest potential genetic differences, while public critics of Western psychiatry point to the overreliance on psychoactive medications (e.g., Whitaker 2001; Comer 2013). But overwhelmingly, discussions point to a "more therapeutic context for recovery" (Bresnahan et al. 2003, p. 29), the "psychosocial environment" (Comer 2013, p. 442), or the "support and demands of the patient's community" (Perkins and Lieberman 2006, p. 5 ). According to Hopper (2003), cultural differences between developing and developed societies stand as the constant but "ghostly" presence for "unexplained variance." Most often, then, the focus on culture as shaped by levels of societal development underlies the WHO schizophrenia paradox.

Thus, for persons with mental illness in the premodern era, Grob (1991) describes the logic of "community stewardship," whereas the modern institutional solution to mental illness became the asylum. In the former, under the logic of the WHO paradox, persons with mental illness remained integrated in their communities. But in the latter, removing individuals from their communities for long periods of time created a new status of 
"mental patient," making these individuals "the other" upon their return to the community, which generated stigma both inside and outside of the treatment system (Goffman 1961, 1963). Even with deinstitutionalization, the stigma of treatment remained (Pescosolido and Rubin 2000; Pescosolido 2013).

Discussions of the WHO paradox tie societal form to life chances for schizophrenia through culture. While the developing/developed dichotomy is used in various ways in the debate, most often this distinction is seen as a "broad and fluid category," grouping countries "characterized by predominant traditional community structures, beliefs, and values" (Kohn, Wintrob, and Alarcon 2009, p. 734). How culture is actually used (Swidler 2001) in the case of mental illness is most directly translated as either support or stigma. Speculations on the mechanisms underlying the WHO paradox have inevitably suggested that the more "traditional" cultures of developing societies neither develop nor endorse prejudicial attitudes and beliefs well documented to exist in modern cultures of developed societies (e.g., Star 1955; Crisp et al. 2000; Angermeyer and Dietrich 2006).

Thus, the irony of the greater individual freedom expected in modern society is greater stigma toward individuals with mental illness. Defined most commonly in contemporary work (Link and Phelan 2001), the stigma of mental illness results from the label as well as the disorder, producing both prejudice (attitudes/beliefs) and discrimination (behavior) that translate into health disparities. As a result, research suggests that the life expectancy of individuals with mental illness is reduced by 7 to 15 years, depending on the country (Miller, Paschall, and Svendsen 2006; Laursen 2011). Further, the effects on personal relationships, unequal medical treatment, job discrimination, and other life spheres are devastating (Corrigan 2012).

Economic development and the cultural myth of public stigma.-Perhaps not surprisingly, the level of development corollary of the WHO paradox aligns with early sociological ideas that Giddens (1976, pp. 71819) labeled the theory of industrial society. Briefly stated, the nature of social life before the transition to industrial society was bound to the community more than the individual, to strength of family ties, and to the greater levels of contact and inclusion among individuals with lives circumscribed locally. Such polar typologies of societal organization are familiar in social theory - status versus contract, gemeinschaft versus gesellschaft, mechanical versus organic solidarity, and concentric versus intersecting circles. As ideal types, societal structures shape and are shaped by cultural dynamics. This is suggested, at least in part, in the sociological tradition in which Simmel (1955, pp. 138, 162), among others, argued that "the number of different social groups in which the individual participates is one of the 
earmarks of culture" that "implies the ideals of collectivism and individualism." In traditional societies, most likely to be described as embedded concentric circles, individuals' networks provide a dense safety net of social supports and lower in-group prejudice. The social cost of greater individual identity and freedom in the transition to industrial society lies in not only the greater predisposition to psychological tensions but also weaker social contact (see Fischer [1982] on the "community lost" hypothesis; also Pescosolido and Rubin 2000). In fact, "contact" has been documented across many studies to have a robust negative association with stigma (see Couture and Penn 2003 for a review).

Yet, how this argument squares with recent widespread contentions that stigma is dissipating in the West may be problematic for the standard stigma-based corollary of the WHO paradox. Widely thought to decrease stigma and increase social integration, the logic of deinstitutionalization began to take hold as early as the 1950s in the West (Brown 1985; Pescosolido et al. 2010). Elsewhere, large institutional care continues to be the norm for treatment. With such contradictory conditions, we follow the lead of the original ISoS researchers by focusing on myth and bringing data to bear. To examine their potential to serve to unravel the WHO paradox, we develop two foundational CMPS propositions. Generally, a simple cultural argument of the WHO paradox corollary can be expressed as:

CMPS Foundational Proposition 1.-Level of economic development is associated with negative cultural schemata and scripts toward persons with mental illness. Specifically, on average, more of the public in developed societies reports stigmatizing attitudes toward and beliefs about persons with mental illness (i.e., a positive association between level of development and percentage stigmatizing).

Cultural ideas passed on through textbook discussions (see list in table A1) of the better prognosis hypothesis and speculation on the underlying mechanisms suggest three specific aspects of cultural beliefs and attitudes at work. Briefly stated, it is commonly argued that cultural scripts in developing societies promote tolerance, inclusion, and support for collective assistance, while those in developed societies produce the opposite.

First, and most specifically, debates and discussion often cite the high level of tolerance in the community (Beardsley and Pedersen 1980, p. 439), arguing that in developing societies, individuals are less judgmental, critical, hostile, or controlling (Rosenberg and Kosslyn 2011; Comer 2013, p. 443). The moral economy, "the cultural trust of deep-rooted principles that guide collective life" (Hopper, Wanderling, and Narayanan 2007, p. 272), is more open to difference and to "odd behavior" (Rosenberg and Kosslyn 2011, p. 553). Under this view, cultural schemata and emotional scripts that reflect negative stereotypes leading to the expression of negative affective re- 
actions or that support forced treatment are held by fewer individuals in developing societies. Similarly, Leff (2001b) argues that less tolerance for symptoms and behaviors associated with schizophrenia are associated with urbanization and industrialization. Consequently, the intolerance propositions (IP) derived from the CMPS focusing on schizophrenia are:

CMPS1 IPA.-More individuals in developed than in developing societies report traditional prejudice toward individuals described with schizophrenia.

CMPS1 IPв.-More individuals in developed than in developing societies report negative affect toward individuals described with schizophrenia.

CMPS1 IPC.-More individuals in developed than in developing societies report support for coercive treatment of individuals described with schizophrenia.

Second, speculation on underlying outcome differences surrounds issues of exclusion in society. While little research, especially outside the West, has examined social interactions in the day-to-day lives of individuals with schizophrenia, individuals in developing societies have more interaction in their communities or are expected to play a functional role in society (Apiquian et al. 2006, p. 161; Rosenberg and Kosslyn 2011, p. 553, respectively). They are more accepted (Beardsley and Pedersen 1980, p. 439; Stroup and Morrissey 2001; Apiquian et al. 2006, p. 161; Glicken 2011, p. 426). In sum, developing societies hold cultural prescriptions that integrate "the mentally ill into the daily life of the family and community" (Kohn et al. 2009, p. 744; Rosenberg and Kosslyn 2011, p. 553). Conversely, in developed societies, the cultural schema changes, rejection increases, and inclusion decreases for individuals with schizophrenia. If the mechanism that underlies the WHO paradox lies in cultural scripts that connect development with more or less inclusiveness and social integration, the implied exclusion propositions (EP) are:

CMPS1 EPA.-More individuals in developed societies than in developing societies report preferences for social distance, overall, as well as in specific social domains of work, family, and community.

CMPS1 EPв.-More individuals in developed societies than in developing societies report preferences for exclusion from social roles that tap into prejudice associated with schizophrenia.

CMPS1 EPC.-More individuals in developed societies than in developing societies report expectations of community rejection toward individuals described with schizophrenia.

Third, an explanation centering on collective cultural orientation sees group needs, cohesion, and interdependence taking precedence over individual autonomy and independence in developing societies. As a number 
of textbook discussions conclude, the WHO paradox likely taps into larger cultural climates that tend to receive more support from family, relatives, and friends (Perkins and Lieberman 2006; Kohn et al. 2009; Rosenberg and Kosslyn 2011, p. 553; Comer 2013). This line of explanation draws directly from social science theories (e.g., Simmel 1955). As societies develop, the cultural emphasis shifts away from the collective to the individual. Openness and community acceptance are replaced by fear of disclosure as well as less support and sympathy. In turn, in the West, the greater emphasis on the individual lowers support for community stewardship and assistance, replaced by greater reliance on individual solutions. Correspondingly, greater public stigma is attached to individuals and families who, for example, use state-based programs for assistance. Specifically, the individualistic propositions (InP) are:

CMPS1 INPA.-More individuals in developed societies than in developing societies report a lack of sympathy, fear of personal and family disclosure, and a need for the family to maintain the secret of mental illness in the family.

CMPS1 INPA.-More individuals in developed societies than in developing societies report rejection of state-based programs to assist persons with schizophrenia.

CMPS1 INPA.-More individuals in developed societies than in developing societies report perceived shame among individuals and their families in accepting state-based assistance to cope with schizophrenia.

Cultural Types of Societies: Beyond the Level of Development Hypothesis Social scientists have had a long, uneasy, and equivocal relationship with the developing/developed dichotomy and modernization theory. Giddens (1976, p. 721) described these conceptualizations as a set of stale ideas that are a residue of the 19th century, arguing for a revitalized comparative sociology. In response, novel ways of categorizing the social organization of societies followed (e.g., Portes 1976; Rueschemeyer, Stephens, and Stephens 1992; Krugman 1997; Meyer et al. 1997; Stephens, Huber, and Ray 1999; Fukuyama 2001).

Perhaps the best known recalibration of these ideas comes from Inglehart's work in the World Values Survey (WVS), where the two dimensions of traditional/secular and survival/self-expression become the basis for important cultural differences that capture the intersection between economic development and path dependence (Inglehart and Baker 2000). Thus, Grief (1994, p. 913) argues that in collectivist societies, members are involved in the lives of others, whereas individualist societies value self-reliance.

This line of inquiry did not escape the attention of those involved in the better prognosis debate. As Rosenberg and Kosslyn (2011, p. 552) suggest, 
"the important distinction may not be the level of industrial and technological development of a country, but how individualist its culture is." Using economic development measures may offer only one test of the cultural context corollary of the WHO paradox. The heart of this second foundational proposition lies in the idea that general cultural climates shape specific cultural attitudes and beliefs toward mental illness and the experiences of people with mental illness. A society that is, for example, generally less tolerant will also inculcate in its members' intolerant attitudes toward individuals with schizophrenia. In turn, people with mental illness will report more stigmatizing experiences. In fact, these ideas have found support for the first time in recent research (Mojtabai 2010; Evans-Lacko et al. 2012). The CMPS proposition targets more specific macrolevel indicators of societal-wide intolerance, exclusion, and individualism. We replace a consideration of level of economic development (LOED) with general cultural scripts of intolerance, exclusion, and individualism:

CMPS Foundational Proposition 2.-Societal levels of intolerance, exclusion, and individualism are associated with negative cultural schemata and scripts toward persons with mental illness. Specifically, more of the public in societies with general climates of intolerance, exclusion, and individualism reports negative attitudes toward and beliefs about persons with mental illness (i.e., a positive association between these general measures of cultural climate and stigma).

Using the same categorization of the trifold nature of stigma, we match three sets of general climate and specific stigma concepts, replacing cultural climate for level of development. We respecify a more tailored set of parallel propositions. More individuals who live in societies characterized by cultural climates of greater intolerance, exclusion, and individualism, compared to those who do not, report:

The Intolerance Propositions

CMPS2 IPA.—-traditional prejudice toward individuals described with schizophrenia;

CMPS2 IPв.- negative affect toward individuals described with schizophrenia; and

CMPS2 IPC.- support for coercion, particularly for treatment, toward individuals described with schizophrenia.

The Exclusion Propositions:

CMPS2 EPA.-preferences for social distance overall, as well as in specific social domains of work, family, and community;

CMPS2 EPB.-exclusion from social roles that tap into prejudice associated with schizophrenia; and

CMPS2 EPC.- expectations of community rejection toward individuals described with schizophrenia. 
American Journal of Sociology

The Individualistic Propositions

CMPS2 INPA. - no sympathy, fear of personal and family disclosure, and need for the family to maintain the secret of mental illness in the family;

CMPS2 INPв.- - rejection of support for state-based programs to assist persons with schizophrenia; and

CMPS2 InPC.--perceived shame among individuals and their families in accepting assistance to cope with schizophrenia.

Previous research efforts.-Attempts have been made to assess the link between stigma and development. In the WHO sites, levels of experienced stigma were high and persistent (Thara and Srinivasan 2000; Hopper, Harrison, Janca, et al. 2007). India had lower levels, but as Hopper and colleagues point out (Hopper, Harrison, Janca, et al. 2007), these data were collected in a later time period, among study families, and in light of the better outcomes documented in the ISoS. However, other preliminary efforts did "not fully support the industrialization hypothesis that is sometimes used to explain the better outcomes of severe mental illness in low-income countries" (Loganathan and Murthy 2008, p. 39). Previous efforts were severely constrained by existing data, producing weak and contradictory results.

\section{DATA, METHODS, AND MEASURES}

Data

Since the World Psychiatric Association's Open the Doors initiative (Sartorius 1997), there has been greater focus on stigma and stigma research across the globe (Kadri and Sartorius 2005; Sartorius and Schulze 2005; Stuart, Arboleda-Florez, and Sartorius 2012; Pescosolido 2013). However, few studies have been population-based, cross-nationally focused, and theoretically and methodologically coordinated. Studies that have met these criteria tend to include only a few countries, are regional, and are focused primarily on Western societies or on individuals with mental illness (i.e., reports of self-stigma; e.g., the 2005-6 Eurobarometer Study; Mojtabai 2010). The SGC-MHS is the first globally targeted, theoretically and methodlogically coordinated empirical examination of the public stigma of mental illness. With support from the U.S. National Institutes of Health (the Fogarty International Center in collaboration with the National Institute of Mental Health and the Office of Behavioral and Social Science Research), the Icelandic Centre for Research, and the University of Ghent, data reported here were collected from representative national samples of individuals in 17 countries.

The SGC-MHS provides the first opportunity to provide a rigorous test of CMPS hypotheses derived from the propositions outlined above using nationally representative samples of respondents in each country. The logistical platform for fielding much of the SGC-MHS was the International 
Social Survey Program (ISSP; see http://www.issp.org), an ongoing annual program of cross-national collaboration among leading social science survey researchers. Initially, ISSP countries were sent electronic invitations from the ISSP secretariat at the time (Tom W. Smith, director, U.S. General Social Survey) indicating the nature of the study and the inclusion requirements (e.g., vignette fielding capability, face-to-face administration, national representation). All countries that indicated a willingness to participate and that fit criteria were included. Additional country-based research teams were added upon their request and were provided technical assistance to meet study standards. In the end, this strategy yielded a remarkable variation of countries geographically (i.e., at least one country on each inhabited continent), developmentally (i.e., high, middle, and low income countries), and politically (e.g., both long-term democracies and postCommunist bloc nations).

Figure 1 provides a global view of the participating countries (with twoletter country abbreviations used in reporting analyses) that provided the basis for the analyses. Each country's total number of respondents and effective sample size for our focus on schizophrenia only are reported. The total number of cases in the SGC-MHS is 18,342, with 6,146 of respondents receiving the schizophrenia vignette.

\section{Sampling and Fielding}

Eligible respondents were noninstitutionalized adults (i.e., age 18 or older). The selection of sample elements for all national cross-sections was based on multistage probability methods. In all countries, face-to-face interviews were conducted by trained interviewers who were closely monitored by survey center personnel who also served as liaisons to the SGC-MHS team for translation, data coding, and preparation and delivery of data files. The overall response rate for the combined SGC-MHS was $65.9 \%$. Institutional review board approval for the current study is held at Indiana University (study 04-9051).

\section{Interview Schedule}

The SGC-MHS interview schedule consisted of two parts: (1) an agreed upon subset of ISSP individual-level sociodemographic variables tailored to country differences (e.g., political, education, and religious systems) and (2) a substantive set of 75 items on mental health and illness including knowledge, attitudes, beliefs, and behavioral predispositions. The challenges of cross-national comparability in the SGC-MHS were addressed in five ways. First, the SGC-MHS instrument began with a revised version of the Problems in Modern Living survey used in the 1996 MacArthur Mental 


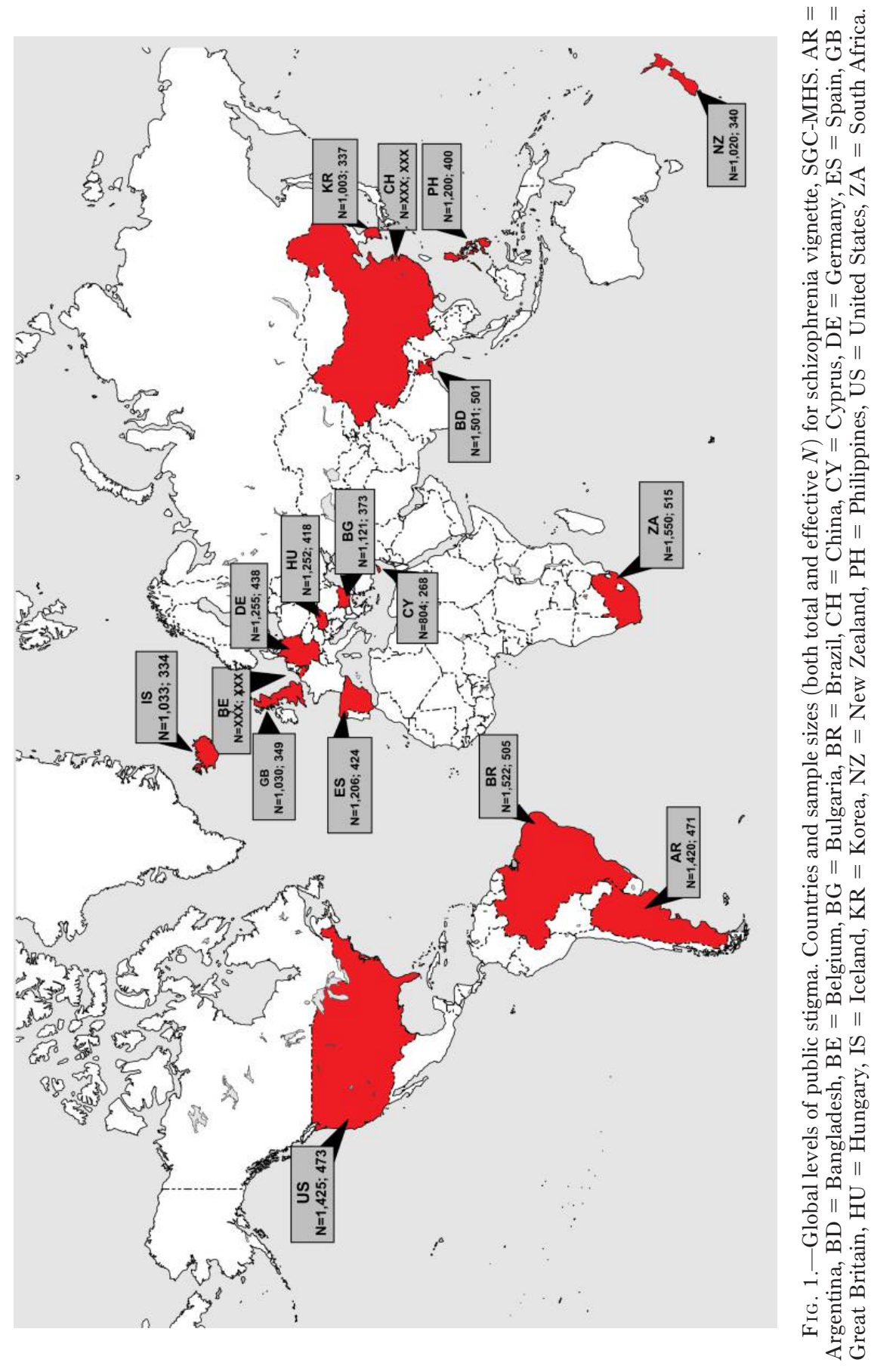

This content downloaded from 131.193.153.188 on April 18, 2016 08:07:04 AM All use subject to University of Chicago Press Terms and Conditions (http://www.journals.uchicago.edu/t-and-c). 
Health Study (Pescosolido et al. 2000). A meeting of survey and mental health experts from the 15 originally participating countries was held in Madrid in 2004 to adapt the instrument for cross-national suitability. In the meeting, mental and physical health vignettes were selected; questions/ response codes were added, eliminated, or changed; guidelines for tailoring to country-specific contexts were developed; and a staggered fielding plan across multiple years was scheduled. Second, an outside psychiatric consultant, involved in the WHO ISoS project, assessed the approach on mental health issues, revised the vignettes, and made final changes during the meeting after participant comments on the new versions were received. Third, a two-step cultural translation process was used that not only required traditional translation-back translation but also incorporated a cognitive interview lasting two to four hours with native speakers not associated with the mental health sector. This was done explicitly to gauge respondent understanding of the questions, provide a sense of socially sensitive questions and the potential for socially desirable response, and glean a layperson's sense of mental illness and treatment in each society. ${ }^{4}$ Fourth, items that embedded cultural variation (e.g., providers available) were collaboratively tailored by SGC-MHS and ISSP teams during the translation phase for each country. Finally, all questions were asked in blocks and in identical order in each country.

\section{Vignettes}

The majority of the SGC-MHS instrument involved assessing respondents' reactions to and evaluations of individuals described in a hypothetical scenario. These vignettes described a person meeting criteria for a Diagnostic and Statistical Manual of Mental Disorders and International Classification of Diseases diagnosis of schizophrenia (depression and control vignette not included here). No labels were offered regarding the scenario. Respondents received one randomly assigned vignette that varied the described person's race/ethnicity (selected by each country to maximize in-group/out-group cleavages through naming conventions) and gender. A series of questions followed referencing the vignette person. The U.S. version of the schizophrenia vignette is presented here: ${ }^{5}$

John/Mary (White)/Tyrone/Shontell (Black) is a White/Black man/ woman. Up until a year ago, life was pretty okay for John/Mary

\footnotetext{
${ }^{4}$ Detail and examples are provided in Pescosolido and Olafsdottir (2008). Manuscript available on request.

${ }^{5}$ Translated versions of all vignettes by country are provided on the SGC-MHS website (http://www.indiana.edu/ sgcmhs/). Also included is a table reporting out-groups and selected names by country.
} 
(White)/Tyrone/Shontell (Black). But then, things started to change. He/She thought that people around him/her were making disapproving comments, and talking behind his/her back. John/Mary (White)/ Tyrone/Shontell (Black) was convinced that people were spying on him/her and that they could hear what he/she was thinking. John/ Mary (White)/Tyrone/Shontell (Black) lost his/her drive to participate in his/her usual work and family activities and retreated to his/ her home, eventually spending most of his/her time on his/her own. John/Mary (White)/Tyrone/Shontell (Black) became so preoccupied with what he/she was thinking that he/she skipped meals and stopped bathing regularly. At night, when everyone else was sleeping, he/she was walking back and forth at home. John/Mary (White)/Tyrone/ Shontell (Black) was hearing voices even though no one else was around. These voices told him/her what to do and what to think. He/ She has been living this way for six months.

\section{Measures}

Public stigma variants: Beliefs about mental illness.-To operationalize stigma, we focus on 33 items tapping several distinctive variants of stigmatizing or prejudicial responses that index the constructs of intolerance, exclusion, and individualistic orientation outlined in the CMPS propositions. ${ }^{6}$ Intolerance indexes commonly held negative attitudes toward persons with mental illness and is operationalized by three public stigma variants: (1) four items assessing traditional prejudice (whether the vignette person is "as intelligent as anyone," "unpredictable," "as productive as others," and "as trustworthy as others"), (2) three items assessing negative affectlemotions (whether the vignette person would make the respondent "feel uncomfortable" or "feel nervous" or is "hard to talk to"), and (3) four items measuring the willingness to use coercive legal means to ensure that the person receives treatment (i.e., "sees a doctor," "uses prescription medications," "goes to a mental hospital," or "is hospitalized").

Exclusion taps the willingness to bar persons from certain social roles and interactions. It is also operationalized by three public stigma variants: (1) six items assessing traditional social distance preferences (i.e., unwillingness to have the vignette person "as a neighbor," "marry into the family," "as a friend," "as a co-worker," or "take care of children" or to "socialize" with the vignette person) and (2) five items measuring role exclusion that

\footnotetext{
${ }^{6}$ Initial analyses indicated that the seven traditional scales from which the items were drawn did not scale in the same way across countries.
} 
assess willingness to deny basic civil rights (i.e., "teach children," "supervise others on the job," "hold public office," "be hired for a job when qualified," and "have children of their own"), all aspects of recovery typically cited as critical (Chatterjee et al. 2008). Finally, (3) a single item assessing community rejection asks whether a person with mental illness "has little hope of being accepted into the community."

Individualism is operationalized by three public stigma variants. First, a series of three items tap into responses that suggest low levels of collective orientation (i.e., low levels of sympathy toward the vignette person, belief that the vignette person should not disclosure his or her condition, and supporting his or her family's decision to keep the situation secret). Second, opposition to state-based responses to the needs of persons with schizophrenia is measured by four items assessing whether the government should "provide jobs," "health care," "education," or "housing" for the vignette person. Third, attitudes toward both individual and family shame in the uptake of benefits are assessed with two items (i.e., whether a person with schizophrenia or members of his or her family should feel shame when accepting governmental assistance).

Within countries, each individual's response to a specific stigma item was coded as a binary variable $(1=$ endorsement of a stigmatizing response, 0 = otherwise; original response categories ranged from 1 to 4 on a Likert scale with no neutral category). On average, missing data did not exceed $11.6 \%$ on any single stigma item. Responses were then totaled to the country level by calculating the item mean for each country (i.e., the proportion providing a stigmatizing response). Additionally, composites of each public stigma variant (i.e., traditional prejudice, negative affect, coercion, social distance, role exclusion, low collective orientation, opposition to state responsibility, and shame) were created by averaging the proportion of stigmatizing responses over the items comprising each variant (the suffix "average" is used in tables and figures). Having both the individual items and a composite measure provides the strongest opportunity for the stigma corollary of the better prognosis hypothesis to find support.

Independent variables: LOED and larger cultural climate.-To evaluate the claim that level of development is positively correlated with public stigma, a set of measures typically used to measure LOED was appended to the country-level SGC-MHS data files from the Penn World Table (2012; see table A2, panel A). For each participating country and each measure, data were coded for the closest year in which the SGC-MHS was completed. Given debates over the best measure, we use a set of measures (table A2, panel A) but report and present graphical findings using only the PPP (purchasing power parity) converted GDP (gross domestic product) per capita, which is known as the Laspeyres index (see Deaton and Heston 2010; Heston, Summers, and Aten 2012). Issues related to the bound- 
ing of the country-level stigma measures between 0 and 1 and the inflation of type 1 error due to multiple hypothesis tests among highly correlated measures (the average Pearson correlation is .96) are discussed in the analytic plan below. Of course, LOED is highly correlated with other measures (e.g., education) often thought to be related to stigma. Yet, studies on both the macro- and microlevel have found, perhaps surprisingly, that sociodemographics are poor predictors of stigma (Schomerus et al. 2012; Pescosolido 2013; Pescosolido and Martin 2015).

Larger cultural climate.-Finding roughly comparable measures across all 17 societies to operationalize the cultural climate measures proved to be difficult. Table A2, panel B, provides details of the measurements and sources. In all cases, we used the year closest to the date of the SGC-MHS fielding in each country. All measures were coded or recoded so that higher scores indicated higher societal levels of intolerance, exclusion, and individualism, to facilitate interpretation. If data were missing, we substituted, where reasonable, the closest earlier or later year.

General cultural intolerance was measured with two items on "freedoms": civil liberties and freedom of the press. The degree of division among groups in the population attempted to quantify general cultural exclusion. These measures included ethnic, language, and religious fractionalization as well as gender inequality and the GINI coefficient. General cultural individualism was operationalized along Inglehart and Baker's (2000) two dimensions of traditional/secular and survival/self-expression. In addition, a measure of private expenditure on health and health care was included.

\section{Analytic Strategy}

For the first research question on cross-national variability, the proportion of respondents in each country providing a stigmatizing response is represented graphically by circles (see fig. 2). The area of the circle is proportional to the value (denoted as the "proportion stigmatizing"; Pescosolido et al. 2013). To gain an understanding of variation in the proportion stigmatizing across countries, the standard deviation for each item is represented through shading. Darker shading indicates greater variation across countries for an item. One-way ANOVAs are used to test whether the proportion stigmatizing varies across countries for each stigma variant.

We use several approaches to test hypotheses on the relationship between stigma and level of development and general cultural climate measures. First, we calculate the Pearson correlation between each measure of level of development (CMPS foundational proposition 1; CMPS P1) and the proportion stigmatizing and between each cultural context measure (CMPS foundational proposition 2; CMPS P2) and the proportion stig- 
Industrial Society and Cultural Schemata

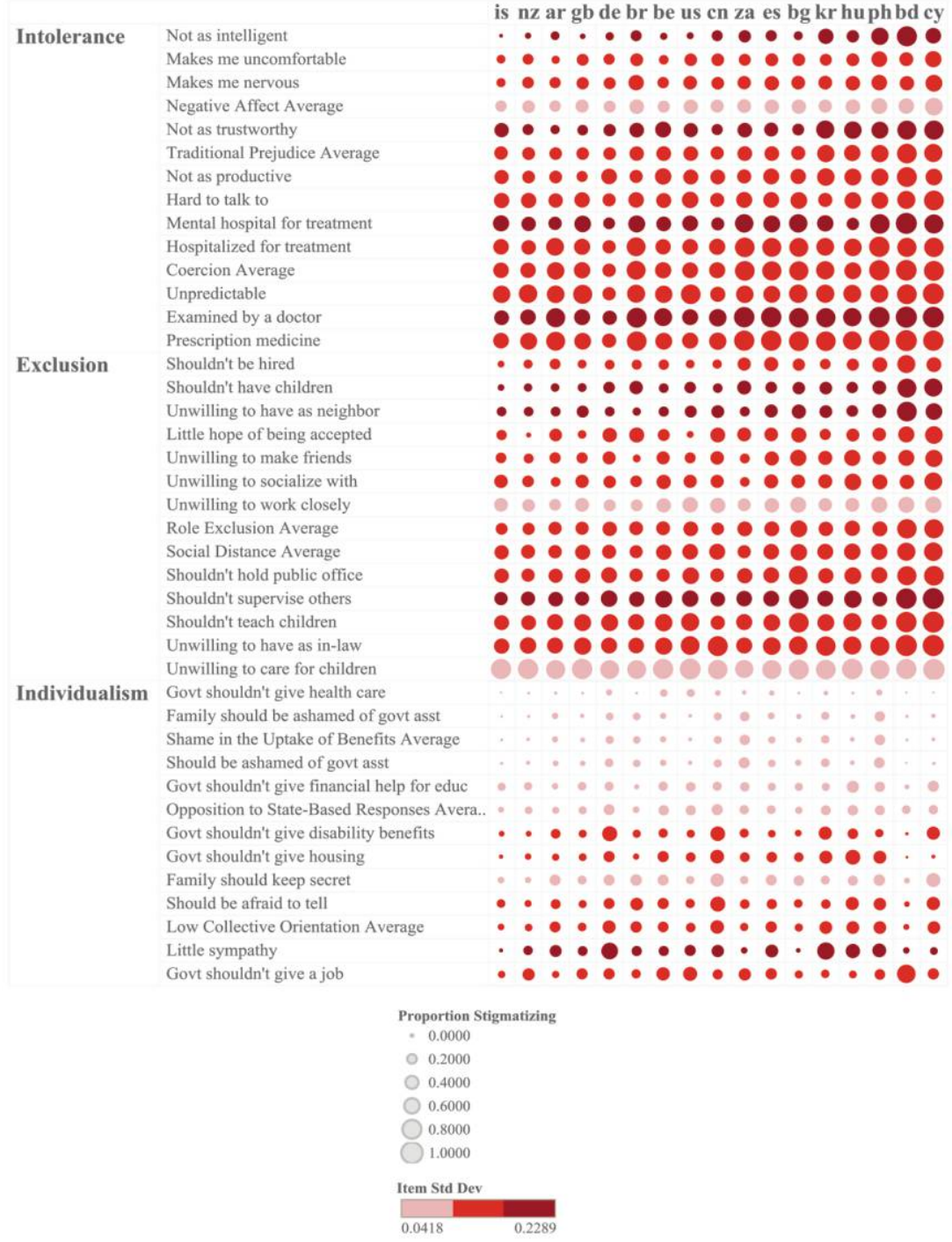

FIG. 2.-Mean and standard deviation of public stigma variants by country, SGCMHS.

matizing, resulting in 492 correlations for CMPS P1 and 410 correlations for CMPS P2. We do this to provide every chance to reject the null hypotheses of no difference. Second, we construct scatter plots for each pair of measures of CMPS P1 and CMPS P2 (i.e., 902 scatter plots). Third, we construct scatter plots for partial correlations that control for LOED in 
the second set to avoid simply replicating earlier findings if cultural context measures are highly correlated with LOED. Scatter plots with fitted regression lines allow a visual examination and a check for outliers, functional form variations, and other distributional anomalies. Individual countries are indicated by the two-letter country abbreviation used in figure 1 .

Because proportions are bounded between 0 and 1 , significance tests based on the normal distribution raise concerns. Therefore, permutation tests based on random shuffles of the data $(N=1,000$ shuffles) are used to examine the null hypothesis of no association (Lehmann and Romano 2005 ). Second, because of the sheer number of correlations, one expects a nontrivial number of "significant associations" (at the $5 \%$ level of significance $)$ merely by chance $(0.05 \times 492=24.6$ for CMPS P1; $0.05 \times 410=$ 20.5 for CMPS P2). We adjust for multiplicity by calculating the expectation of finding significance "by chance" using the false discovery rate (FDR; Benjamini and Hochberg 1995). ${ }^{7}$

To facilitate presentation, we provide density plots of the 492 correlations for CMPS P1 and the 410 correlations for CMPS P2 as well as an illustrative selection of scatter plots (figs. $3-7$ ). The lines at \pm 0.48 for CMPS $\mathrm{P} 1$, at $\pm 0.496 / 0.495$ for CMS P2, and $\pm 0.477 / 0.489$ CMPS P3 adjusted for LOED represent the average of the correlations that demarcate the 05 cutoff region using the permutation distribution. Full correlational analyses are available on request. ${ }^{8}$

\footnotetext{
${ }^{7} P$-values are calculated via permutation tests as follows. Let $\left(x_{1}, \ldots, x_{17}\right)$ be the 17 countries' responses on stigma (i.e., percentage of sample respondents who responded with a "stigma" response), and let $\left(y_{1}, \ldots, y_{17}\right)$ denote the 17 countries' scores on one of the LOED variables. The Pearson correlation is calculated the usual way; however, the $x_{i}$ 's $r^{*}=\sum_{i=1}^{17}\left(x_{i}-\bar{x}\right)\left(y_{i}-\bar{y}\right) / \sqrt{\sum_{i=1}^{17}\left(x_{i}-\bar{x}\right)^{2} \times \sum_{i=1}^{17}\left(y_{i}-\bar{y}\right)^{2}}$ are percentages bounded by 0 and 100 , so the $P$-value calculated from the traditional hypothesis test of $H_{0}: \rho=0$ would not apply. A nonparametric $P$-value is computed by generating a null distribution as follows. We permute the $x$ 's and the $y$ 's and calculate $r$ using the permuted $x$ 's and $y$ 's. We expect no association between the permuted variables, which are now completely disassociated. We repeat this permutation exercise 1,000 times to generate a null distribution of $r$. The $P$-value for $H_{0}: \rho=0$ is calculated as the percentage of the permuted $r$ values that exceed $r^{*}$ in magnitude. For example, if $r^{*}=$ .65 and only 20 of the 1,000 permutation-based $r$ values exceed .65 in magnitude $(>.65$ or $<-.65)$, then the estimated $P$-value is $20 / 1,000=.020$. To minimize the family-wise error rate, we considered the Bonferroni correction. However, while this criterion may be too stringent, some adjustment for multiplicity is in order. We thus used the Benjamini-Hochberg FDR, which ensures that the expected proportion of false significances does not exceed a prespecified level (here, $5 \%$ ). The procedure identifies at least as many, and usually more, associations than does the Bonferroni correction.

${ }^{8}$ Substantive findings reported here do not differ substantially from findings obtained using weights to account for unequal probability of being included in each of the national samples.
} 


\section{FINDINGS}

\section{The Global Landscape of Public Stigma}

Our first research question is descriptive: Is there variation in stigmatizing responses across countries? In figure 2, rows of stigma items and composite measures are sorted by the intolerance, exclusion, and individualism constructs that guided the operationalization of the dependent variables. The item grand mean (the mean proportion stigmatizing calculated across countries) is used to sort the items from top to bottom by increasing public stigma for each construct. The country grand mean (the mean proportion stigmatizing calculated across items) is used to sort the countries from left to right by increasing stigma. Thus, larger (a higher percentage endorsing) circles indicate greater prejudice, while darker circles (higher standard deviation) indicate greater variability in item response.

Figure 2 suggests variation. First, there are cross-national differences in public stigma responses. As indicated by the larger area of the circles, Bangladesh, Cyprus, Korea, Hungary, and the Philippines stand out as having a higher proportion of individuals providing stigmatizing responses. Looking at item variability across countries, "not as intelligent," "not as trustworthy," "mental hospital for treatment," "examined by doctor," "shouldn't have children," "unwilling to have as neighbor," "shouldn't supervise others," and "little sympathy" stand out as having the most variability as expressed by the darker shading of the circles associated with these items. Second, considering the three groupings of constructs (intolerance, exclusion, and individualism), public stigma is both low and less variable when considering items within the individualistic construct - the circles in the individualism panel are small, and the item shading is light. In contrast, the largest circles and items with the most variability across countries are found within the intolerance construct (intolerance panel) and to a lesser degree the exclusion construct (exclusion panel) — the circles are larger and the shading is darker.

Table 1 reports the correlations relevant to hypotheses derived from CMPS P1. LOED is only occasionally associated with public stigma. Using a single "best" measure of LOED, nine correlations are statistically significant. With regard to the three stigma constructs, there are no significant associations with stigma variants tapping into ideas of individualism/collectivism (table 1, panel C). For exclusion, no correlations indicate an association between preferences for LOED and social distance (panel B). Finally, LOED is primarily associated with the coercion measures (panel A).

Overall, using all the diverse measures of LOED, only 108 of the correlations are statistically significant using the permutation solution, reduced to 95 when adjusting for multiplicity using the FDR. Thus, $22 \%$ of the correlations, in the most conservative case to reject the null hypothesis 
TABLE 1

Correlations between Proportion Stigmatizing and Level of Economic Development, SGC-MHS

\begin{tabular}{|c|c|}
\hline & Correlatio \\
\hline \multicolumn{2}{|l|}{ A: Intolerance: } \\
\hline \multicolumn{2}{|l|}{ Traditional prejudice (People like vignette person are . . .): } \\
\hline 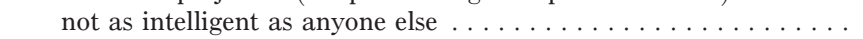 & $-.66^{*}$ \\
\hline 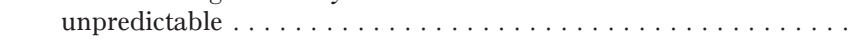 & -.05 \\
\hline not as productive as most other workers $\ldots \ldots \ldots \ldots \ldots$ & -.30 \\
\hline not as trustworthy as anyone else $\ldots \ldots \ldots \ldots \ldots \ldots$ & -.21 \\
\hline Traditional prejudice average $\ldots \ldots \ldots \ldots \ldots \ldots \ldots$ & -.41 \\
\hline \multicolumn{2}{|l|}{ Negative affect (People like vignette person . . . ): } \\
\hline are hard to talk to $\ldots \ldots \ldots \ldots \ldots \ldots \ldots \ldots \ldots$ & -.27 \\
\hline would make me feel uncomfortable $\ldots \ldots \ldots \ldots \ldots \ldots \ldots$ & -.44 \\
\hline would make me feel nervous . . . . . & $-.50 *$ \\
\hline Negative affect average . . . . . . . & -.44 \\
\hline \multicolumn{2}{|l|}{ Coercion (People like vignette person should be forced by law to . . .): } \\
\hline be examined by a clinic or by a doctor $\ldots \ldots \ldots \ldots \ldots \ldots$ & $-.75^{*}$ \\
\hline take medication prescribed by a doctor $\ldots \ldots \ldots \ldots \ldots$ & $-.75 *$ \\
\hline be hospitalized for treatment $\ldots \ldots \ldots \ldots \ldots \ldots \ldots$ & $-.73 *$ \\
\hline go to a mental hospital for treatment $\ldots \ldots \ldots \ldots \ldots \ldots \ldots$ & -.41 \\
\hline Coercion average $\ldots \ldots \ldots \ldots \ldots \ldots \ldots \ldots \ldots \ldots \ldots$ & $-.70 *$ \\
\hline \multicolumn{2}{|l|}{ B: Exclusion: } \\
\hline \multicolumn{2}{|l|}{ Social distance (I would be unwilling to . . .): } \\
\hline have vignette person as a neighbor ...... & -.39 \\
\hline spend time socializing with vignette person $\ldots \ldots \ldots \ldots \ldots$ & .03 \\
\hline have vignette person care for my children/children I know ..... & .41 \\
\hline make friends with vignette person $\ldots \ldots \ldots \ldots \ldots \ldots \ldots$ & -.27 \\
\hline work closely with vignette person on a job $\ldots \ldots \ldots \ldots \ldots$ & -.16 \\
\hline have vignette person marry someone related to me $\ldots \ldots \ldots \ldots$ & -.34 \\
\hline 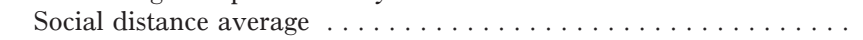 & -.22 \\
\hline \multicolumn{2}{|l|}{ Role exclusion (People like vignette person . . .): } \\
\hline should not be allowed to hold public office . . & -.26 \\
\hline should not be allowed to have children $\ldots \ldots \ldots \ldots \ldots \ldots$ & $-.52 *$ \\
\hline should not be hired $\ldots \ldots \ldots \ldots \ldots \ldots \ldots \ldots \ldots$ & $-.61 *$ \\
\hline should not be allowed to teach children $\ldots \ldots \ldots \ldots \ldots \ldots$ & -.13 \\
\hline should not be allowed to supervise others at work $\ldots \ldots \ldots \ldots$ & -.29 \\
\hline Role exclusion average $\ldots \ldots \ldots \ldots \ldots \ldots \ldots \ldots \ldots \ldots \ldots \ldots$ & -.41 \\
\hline \multicolumn{2}{|l|}{$\begin{array}{l}\text { Community rejection (A person like vignette person . . .): } \\
\text { has little hope of ever being accepted as a member of his/her }\end{array}$} \\
\hline 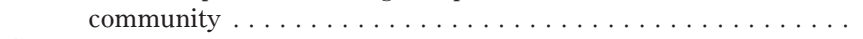 & $-.65 *$ \\
\hline \multicolumn{2}{|l|}{ C: Individualism: } \\
\hline \multicolumn{2}{|l|}{ Low collective orientation: } \\
\hline \multicolumn{2}{|l|}{ I feel little sympathy for people that seem to have a serious } \\
\hline \multicolumn{2}{|l|}{ Vignette person should feel afraid to tell others about } \\
\hline 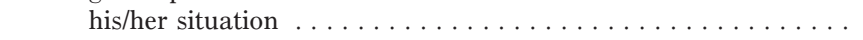 & -.28 \\
\hline \multicolumn{2}{|l|}{ Vignette person's family would be better off if person's } \\
\hline situation kept a secret $\ldots \ldots \ldots \ldots$ & -.29 \\
\hline Low collective orientation average & -.17 \\
\hline \multicolumn{2}{|l|}{$\begin{array}{l}\text { Opposition to state-based responses (It shouldn't be the govt's } \\
\text { responsibility to provide ....): }\end{array}$} \\
\hline a job for name if name wants one $\ldots \ldots \ldots \ldots \ldots \ldots$ & -.06 \\
\hline health care for name $\ldots \ldots \ldots \ldots \ldots \ldots \ldots \ldots$ & .38 \\
\hline housing for name if name can't afford it $\ldots \ldots \ldots$. & -.10 \\
\hline
\end{tabular}




\begin{tabular}{|c|c|}
\hline & Correlation \\
\hline financial help for education if name can't afford it . & .17 \\
\hline disability benefits for name $\ldots \ldots \ldots \ldots \ldots \ldots$ & -.02 \\
\hline Opposition to state-based responses average $\ldots \ldots \ldots \ldots \ldots$ & .01 \\
\hline \multicolumn{2}{|l|}{ Shame in the uptake of benefits: } \\
\hline \multicolumn{2}{|l|}{ Vignette person should be ashamed to receive } \\
\hline governmental assistance $\ldots \ldots \ldots \ldots \ldots \ldots$ & -.37 \\
\hline \multicolumn{2}{|l|}{ Vignette person's family should be ashamed if person } \\
\hline receives governmental assistance $\ldots \ldots \ldots \ldots \ldots$ & -.47 \\
\hline Shame in the uptake of benefits average . . & -.42 \\
\hline
\end{tabular}

Note.-PPP converted GDP per capita (Laspeyres), derived from growth rates of consumption, government spending, and investment at 2005 constant prices. $N=17$. Significance uses two-tailed test using permutation method

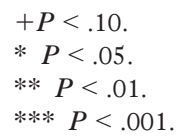

of no correlation, are statistically significant. In fact, the density plot of all 492 sample correlation coefficients in figure 3 shows no correlation supporting the CMS hypotheses. Most correlations are not significant, and, among those that are, the data contradict the idea that the cultural climate of stigma toward schizophrenia is better in lower or middle income countries.

Counter to predictions in the specific CMPS-derived hypotheses, the direction of the relationship between development level and public stigma

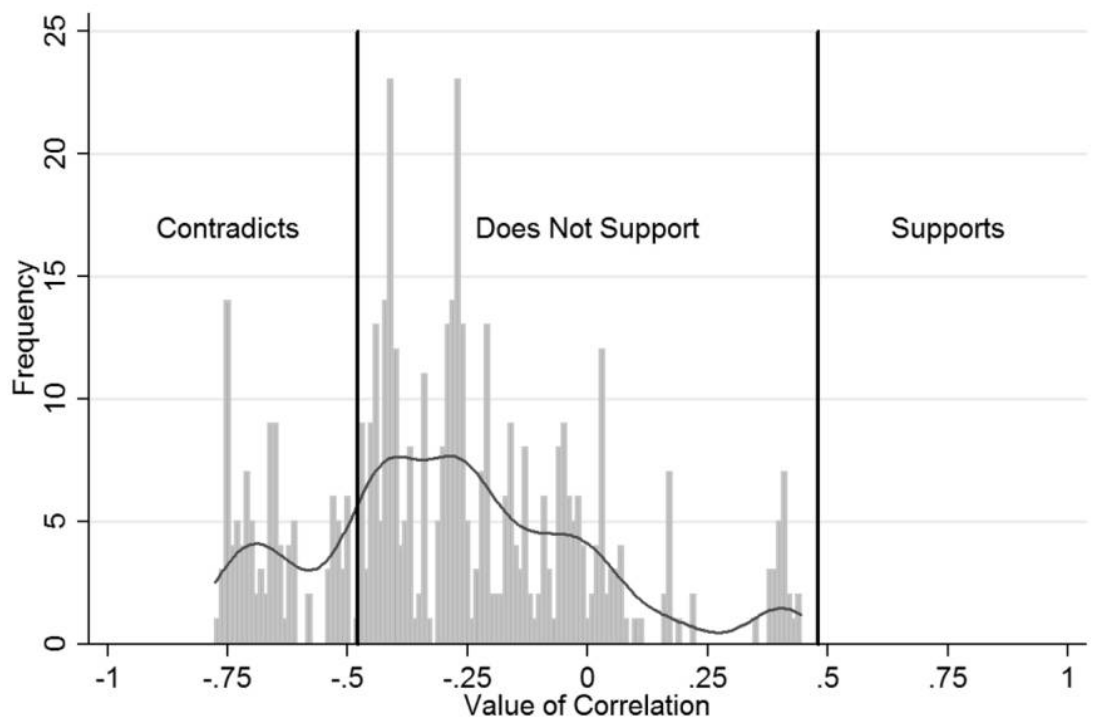

FIG. 3.-Density plot of public stigma and LOED correlations, SGC-MHS 


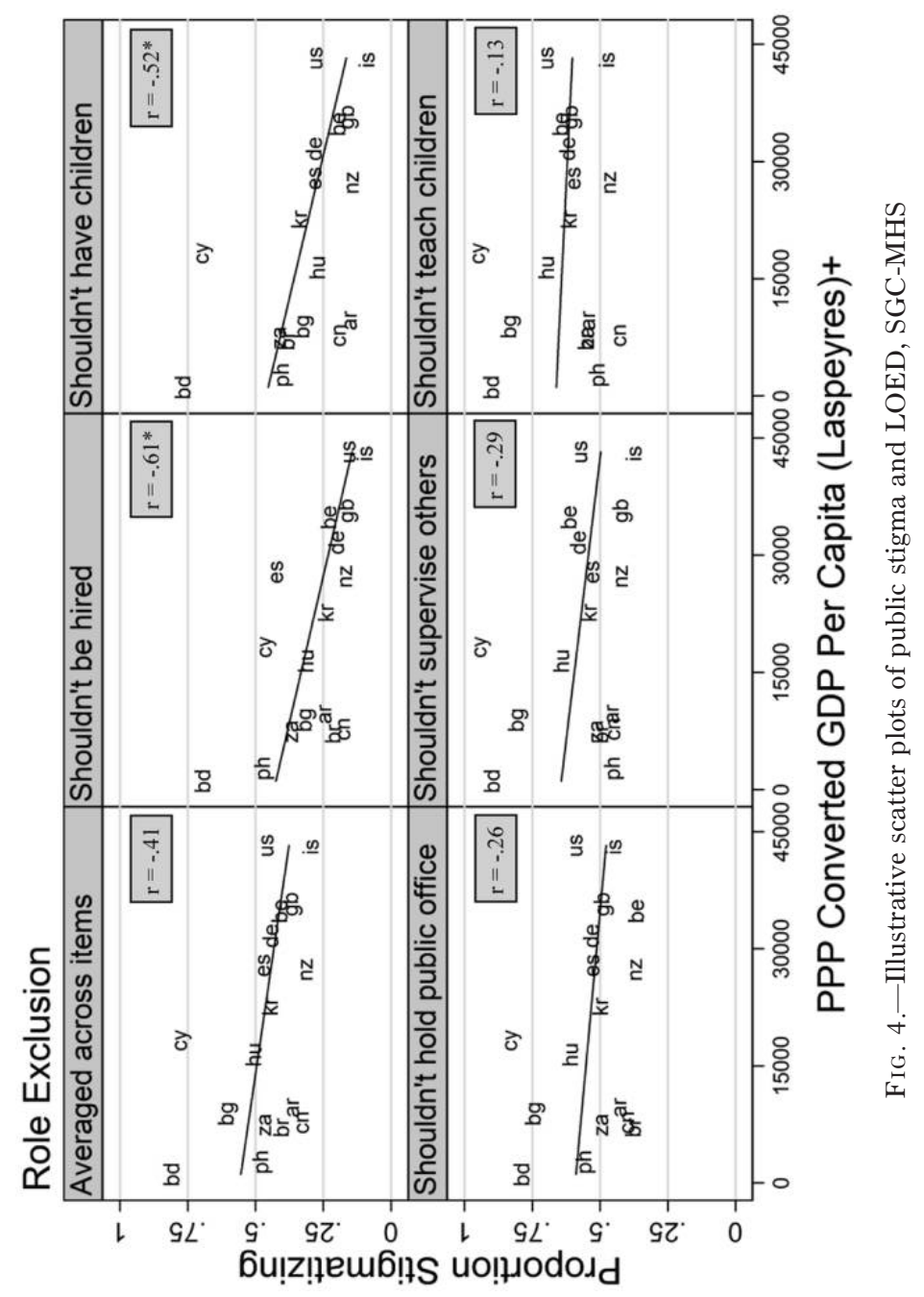

This content downloaded from 131.193.153.188 on April 18, 2016 08:07:04 AM All use subject to University of Chicago Press Terms and Conditions (http://www.journals.uchicago.edu/t-and-c). 
is overwhelmingly negative. That is, levels of stigma are generally higher in "less" well-developed countries than in "more" developed countries. For example, figure 4 illustrates the findings on exclusion using the set of role measures. The scatter plots for the averaged measure of role exclusion prejudice and separately for the five measures that comprise it are displayed. Individuals in the low income countries of Bangladesh, Philippines, South Africa, Argentina, Brazil, and China report, on average, that people like the one described in the schizophrenia vignette should not be hired or have children. Middle income countries like Hungary, Cyprus, and Korea report, on average, less stigma, while high income countries like the United States, Iceland, Belgium, and Great Britain report the lowest levels of stigma.

\section{CMPS Proposition Set 2: General Cultural Context and Public Stigma}

To evaluate whether the cross-national variation in stigma toward schizophrenia is systematically patterned by the larger climate of intolerance, exclusion, and individualism, we drew from measures of the larger cultural climate of intolerance, exclusion, and individualism, arguing that such general cultural scripts may shape specific cultural scripts for schizophrenia. The results here are quite different. Figure 5 displays density plots for both bivariate correlations and partial correlation between larger cultural context measures and the specific measures of stigma toward schizophrenia. In the bivariate case, of the 410 correlations computed, 60 correlations were significant, providing support for the idea that more intolerant, exclusionary, and individualistic cultures are associated with stigma. Of these, 32 support the CMPS proposition that negative cultural contexts produce higher percentages of individuals reporting stigma (eight are significant once the FDR correction was applied), while 28 contradict this notion (none remain significant after FDR correction was applied).

Again, however, even results that appear to support the CMPS proposition do not support ideas about level of development as a key factor. There are two patterns. The first repeats the earlier results of lower public stigma among more developed countries. Countries that spend more on private health care, for example, do report higher average rates of public stigma (e.g., role exclusion), but those countries are not only those considered to be less developed (fig. 6). Whether or not LOED is controlled, countries as dissimilar as the United States and Cyprus, where the percentage of health expenditures are more likely to be private, also have more individuals reporting stigma. ${ }^{9}$ Perhaps the most robust findings in this

${ }^{9}$ We correlated the residuals of both $x$ (e.g., private health spending) and $y$ (e.g., social distance average) on $z$ (i.e., LOED). 
American Journal of Sociology
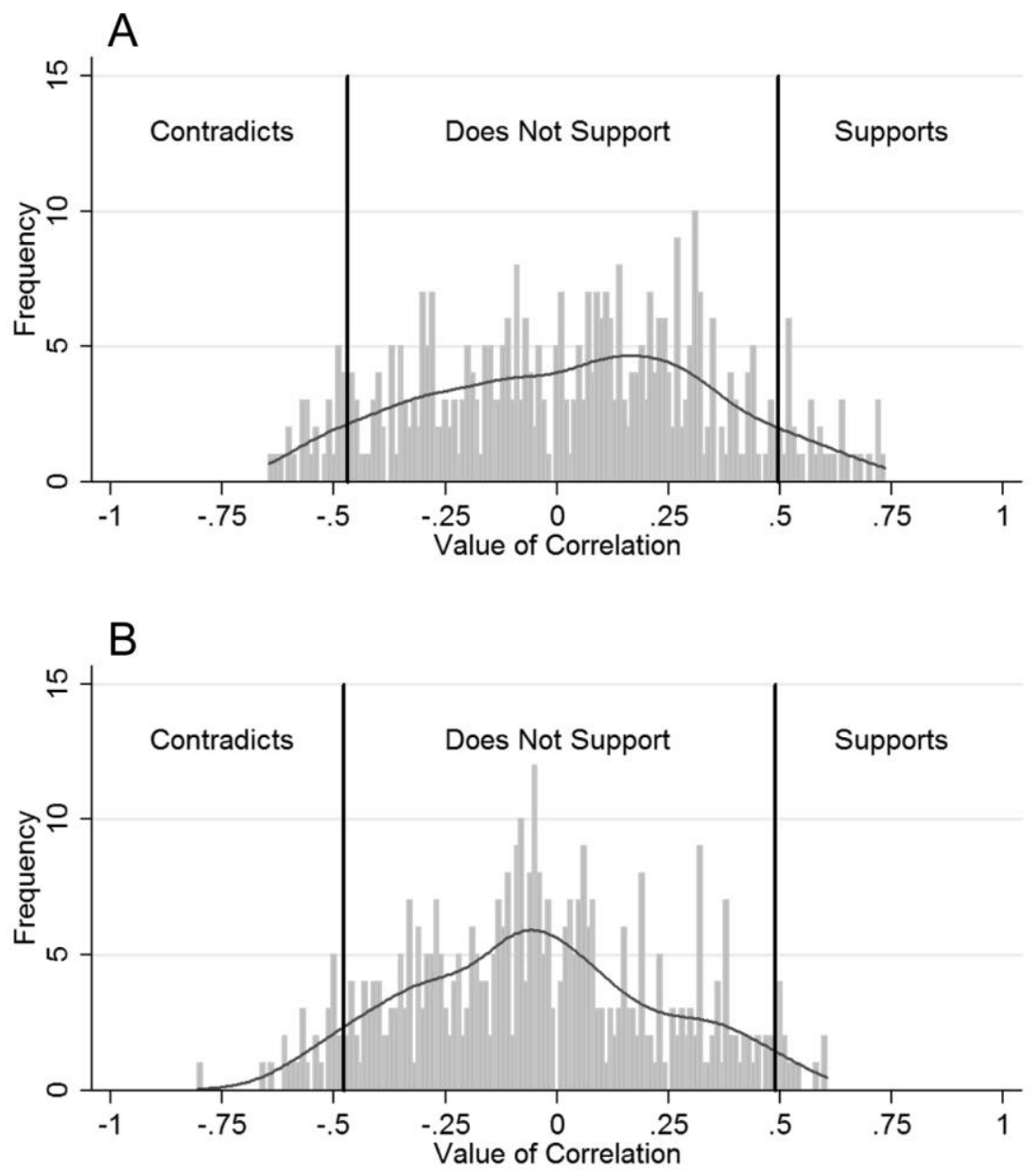

FIG. 5.-Density plot of unadjusted and adjusted public stigma and general cultural context, SGC-MHS. $A$, Unadjusted correlations; $B$, adjusted corrections.

analytic section are associated with two sets of measures that tap into the collectivist versus individualist dimensions. Overall, the Inglehart and Baker (2000) measures suggest that more "traditional" (vs. "secular") and "survival" (vs. "self-expression") oriented societies have higher reported rates of stigma. Those WVS "traditional" countries include those considered to be both high and low along the economic development continuum. Again, then, there appears to be no clear correspondence with level of development (figs. 6 and 7).

In sum, larger cultural context may matter, as sociologists have long held. Yet, how it matters and what this means fundamentally challenges the 


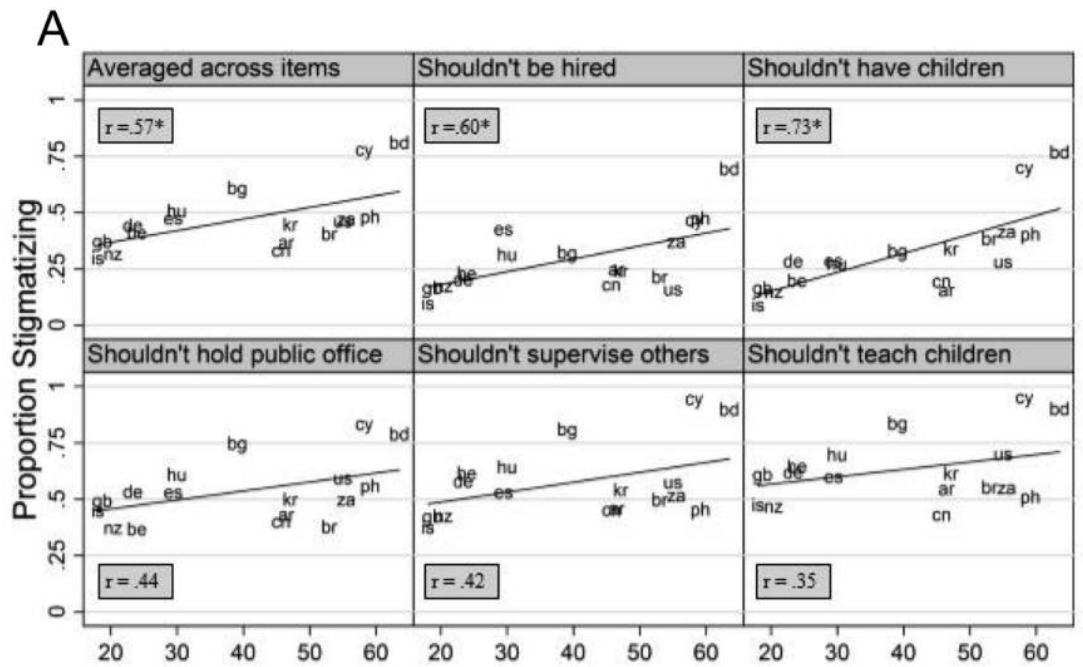

Private health expenditures as a \% of total health expenditures+

B

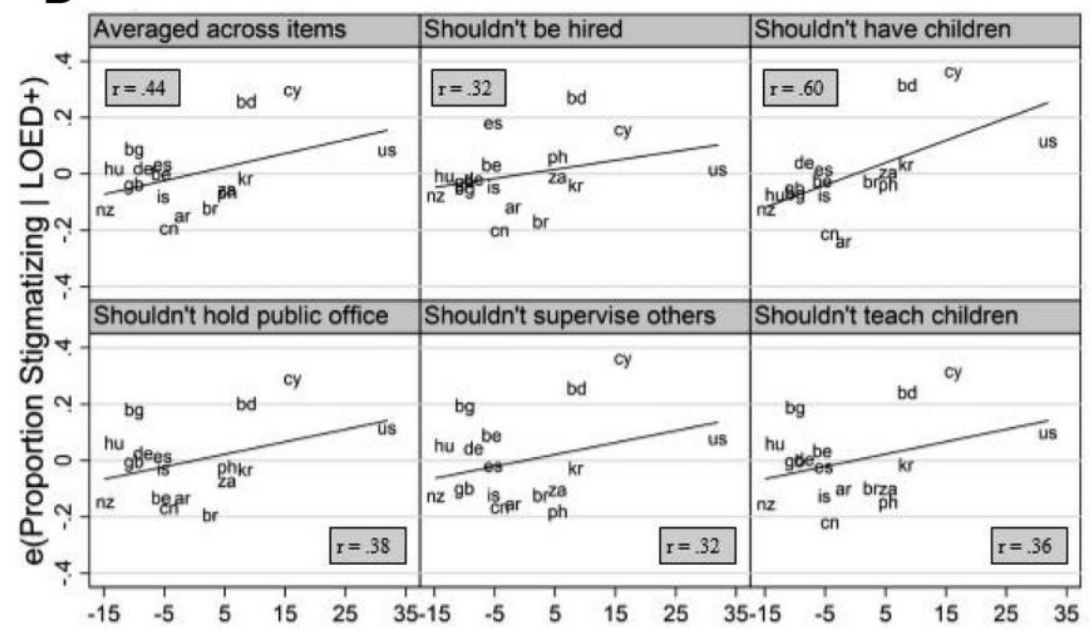

$\mathrm{e}$ (Private health expenditures as a \% of total health expenditures | LOED+)

FIG. 6.-Illustrative scatter plots of public stigma and general cultural climate, private health expenditures, unadjusted $(A)$ and adjusted $(B)$ for LOED, SGC-MHS. 

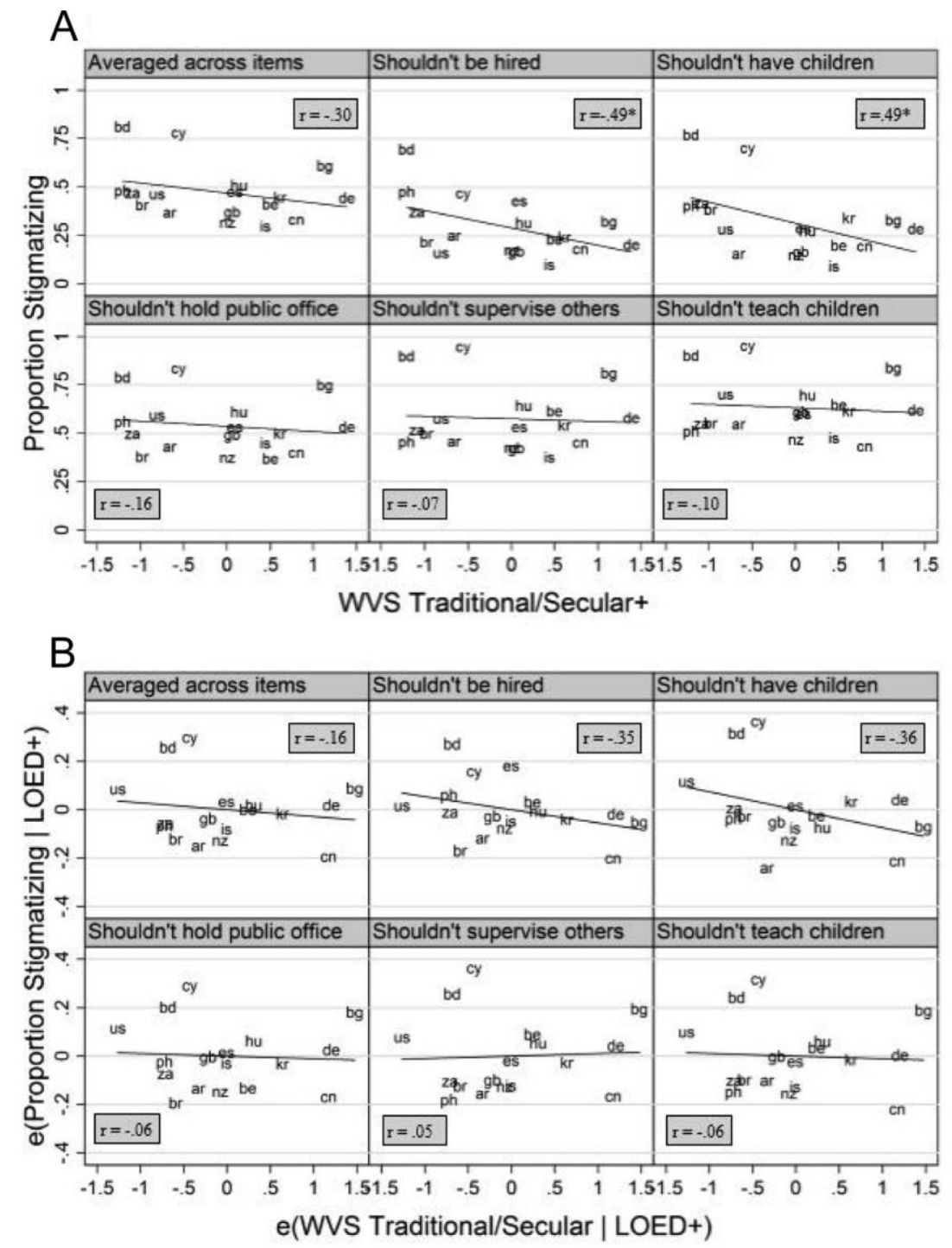

FIG. 7.-Illustrative scatter plots of public stigma and general cultural climate, WVS measure of traditional/secular culture, unadjusted $(A)$ and adjusted $(B)$ for LOED, SGC-MHS, 
widely held corollary of the WHO paradox. These findings require a basic and more complex rethinking of the multiple layers of social structures and their relevance in terms of content and social interaction (Pescosolido et al. 2008).

\section{DISCUSSION AND CONCLUSION}

Thus, the creation of groups and associations in which any number of people can come together on the basis of their interest in a common purpose, compensates for that isolation of the personality which develops out of breaking away from the narrow confines of earlier circumstances.

(Simmel, The Web of Group Affiliations)

Our concern with the global landscape of stigma came from a confluence of two issues. First, when the World Psychiatric Association's Global Program against Stigma and Discrimination because of Schizophrenia (Sartorius 1997) called for both stigma research and stigma reduction efforts, there had been few nationally representative studies, making it difficult, if not impossible, to understand how the stigma of mental illness varies across countries. As sociologists, we were in a unique position to begin to close that gap. Second, the WHO paradox raised the issue of a patterned relationship between stigma and levels of development. If, in fact, the global distribution of public stigma could be mapped to claims of outcome heterogeneity, documenting these differences could offer a scientific wedge into programs to decrease stigma's negative impacts. In sum, while the potential utility of cross-national comparisons was compelling, the extant database was thin. In fact, reconsideration of the common beliefs associated with the WHO paradox, including those about public stigma, has been called an "imperative" (Shrivastava and Johnston 2010). To that end, we focus on one aspect of what Edgerton and Cohen (1994) call the "black box of culture," the larger cultural climate in which individuals, their family members, and providers experience and respond to serious mental health problems.

We asked three questions: Is there cross-national variation in how the public responds to persons described as having a symptom set consistent with Diagnostic and Statistical Manual of Mental Disorders and International Classification of Diseases diagnoses of schizophrenia? The data in the SGC-MHS support an answer of yes. Second, we asked whether that variation is consistent with the LOED as articulated in the most common corollary of the WHO paradox. Our analysis contradicts this assertion: the answer is no. There is no support for the idea that more traditional societies, those characterized by lower levels of economic development, have less stigmatizing cultural contexts. In fact, where there are associations between levels of development and public stigma as measured by items 
that tap into intolerant, exclusionary, and individualistic attitudes toward individuals described as meeting psychiatric criteria, the findings are the opposite. Countries like Iceland, Germany, and the United States report the lowest levels of public stigma. Third, do countries that have cultural attitudes and behavioral predispositions that can more generally be characterized as intolerant, exclusionary, and individualistically oriented also report more negative cultural schemata toward persons with schizophrenia? Our analyses suggest a more complicated answer. There is support for and against the hypothesis. However, what is evident in the results is that there is no support for the idea that the "traditional" societies of Bangladesh or the Philippines are among those with lower proportions of adults who express stigma. In fact, it is those societies that are more traditional (vs. secular) and hold more values emphasizing survival (vs. self-expression) that report greater stigma at the contextual level.

Thus, the SGC-MHS was designed to ask whether there are national cultures that can be characterized as less stigmatizing. The answer appears to be yes. The data suggest substantial differences in how individuals in different countries respond to a scenario that described a person meeting criteria for schizophrenia. However, are these the "developing" societies? The answer is no. The variation in national responses appears to have little to do with levels of development or national wealth. Although not consistent, there is some suggestion that public stigma may be lower in countries with higher levels of GDP, but there is no evidence to support the claim that public stigma is lower in less developed countries.

How do these findings relate to general theoretical ideas about societies and their cultural schemata? The quotation above from Simmel provides part of the answer. What comes with modern society is a greater focus of the individual. And with that greater individuation comes more concern, perhaps especially in the postmodern era, with the focus on the self. Our development of the dual set of CMPSs and testing hypotheses relevant to the level of development corollary corresponds to Giddens's (1976, p. 703) discussions of the theory of industrial society as leading interpretations and of widely held views, which are either false or highly misleading (Giddens 1972 , p. 357). Curiously, we are led to the same set of issues and concerns he discusses (Giddens 1976, p. 715) on how "the false images of the past which they have fostered have had an unhappy influence on the contemporary debate."

Where does this leave us in thinking about culture in different societal structures? Modern extensions of Simmel suggest that we move from understanding the historical "stages" of society to thinking about the existence of coexisting types of cultures and structures that are more or less predominant in any time and place (Pescosolido and Rubin 2000). As Greif (1994, p. 913) points out, "any society has individualistic and collectivist 
elements, and categorization is a matter of their relative importance" (see also Bellah et al. 1985). Contemporary sociology has also become more explicit about the reinforcing or opposing levels of social life and social organization. If it is indeed the case that some important outcomes are better in India than in the Western European welfare states, then it must be that the organization of care and the "small worlds" (Fischer 1982) in which individuals live provide a better shield against the negative cultural schemata that exist there. On the individual level, across all WHO ISoS centers, one of the best predictors of a poor outcome was social isolation (see n. 2).

As one of the primary analysts of the WHO data has concluded, what the WHO ISoS studies may suggest to us is that "the stigma of MI is not so much undone as surmounted" (Hopper, Harrison, Janca, et al. 2007, p. 273). Culture as an "institutional armature and internalized program for collective living" is related to "exculpatory beliefs, less complex cognitive demands, extended family support, [and] accommodating work regimes, absent stigma" (Hopper 2008, p. 203). Modern psychiatry in Western societies is built on a very individualistic model of treatment with only occasional programs that intensely involve family or the community, even as research has clearly documented that such an orientation has been proven effective in reducing the relapse rate of schizophrenia (Leff 2001a; Lauronen et al. 2007).

We do not need to dismiss the link between macrolevel culture and microlevel life chances. The findings that support the impact of larger cultural context on public stigma do not align with broad ideas of "development" that dominate the better prognosis hypothesis. Rather, we need to look toward a different and complex set of ideas of what about cultural climate matters and how they may interact with local social networks. As Hopper and his colleagues note, we need to understand stigma not as "unmoored" but in terms of "the cultural matrix to locate its meaning and force" (Hopper, Harrison, Janca, et al. 2007, p. 272).

Thus, rather than the larger societal context, family or the nature of treatments received has been cited (Menon and Shankar 1993; Kim Hopper, personal communication). Yet, it is critical to remember that these issues are not unrelated. As Hopper, Harrison, Janca, et al. (2007) note, the WHO studies showed better outcomes in two sites in India, not in the country as a whole. The WHO study sites are not funded by the WHO; they are selffunded and carried out by volunteers. Many sites in the developing world, in particular, are located in leading academic psychiatric facilities or cuttingedge, model programs. Local social networks in treatment, in families, and in the interaction of community and health care systems can override the larger cultural context. The idea that social ties can stand in opposition to or operate differently in different macrocontexts is an important element in sociological approaches (e.g., Kadushin 1983; Pescosolido 2006). 
American Journal of Sociology

\section{Limitations}

We consider five significant limitations to these findings. First, the SGC-MHS relied on the ISSP platform, which we saw as an advantage for valid national comparisons. It has disadvantages, however, including the restricted set of countries (particularly low income countries) that participated. And in particular, the lack of survey research capacity among African nations represents a serious limitation that may be overcome as research infrastructure building continues to be supported. In addition, the SGC-MHS's 17 countries and the WHO ISoS's 10 countries are not the same. Yet, fielding efforts continue to be challenging in lower income countries.

Second, the nature of the SGC-MHS (primarily the requirement to use vignettes) prohibited the inclusion of countries (whether high, middle, or low income) with greater land mass and uneven population distributions (e.g., Canada, Australia) that made face-to-face interviews unfeasible. Yet, the vignette approach was critical for theoretical (i.e., no labeling) and methodological (i.e., literacy) issues (Pescosolido 2013). With one "case" description that underplays the spectrum of schizophrenia, we cannot gauge how different the national profiles would have been if labels were attached, if treatment had been described, or if chronicity had been varied.

Third, even with 17 collaborating countries, the ability to determine which national characteristics are in operation is limited. Fourth, Hopper and Wanderling (2000, p. 842) suggest that the WHO paradox is so robust that it can be "taken as prima facie evidence for the relevance of 'culture.'" They are skeptical about whether a structured approach like the SGCMHS can actually tap into their conceptualization of culture as life worlds. Ruling out one level of analysis, public stigma, does not allow a conceptualization of the multiple layers of society that may reinforce or counter one another. This either-or approach may not serve the growing acceptance of complexity as a fundamental characteristic of social systems (Ostrom 2009).

Fifth, the profound effects of stigma that hold the potential to affect individual, family, and societal institutions are experienced in behavior, not necessarily in attitudes. However, as we have discussed elsewhere, attitudes may represent the litmus test, since scientific evidence suggests that individuals are more willing to express prejudice than to act on it in real life situations (Pescosolido 2013). A more relevant limitation of our findings refuting the better prognosis hypothesis, however, might be found in the nature of the attitudes assessed. As is well known, the measurement of socially unacceptable attitudes (e.g., prejudice) is frequently associated with a tendency of respondents to provide the politically correct or socially desirable answers to the questions asked (Dillman 1978). Particularly in the current case, organized efforts on the part of advocacy groups, governments, and the media have been mounted to educate the public as to the 
causes and consequences of mental disorders. In other words, as a result of exposure to mental health awareness media campaigns, particularly in more developed nations, respondents may have been conditioned to provide less stigmatizing answers. If true, this argument might cast doubt on the findings of the current research.

While plausible, this argument, particularly regarding attitudes toward people with schizophrenia, seems doubtful. There is little, if any, solid empirical evidence that the stigma associated with schizophrenia has lessened in recent years. For example, a 2007 report published by Mental Health in America indicates that $55 \%$ of Americans report feeling uncomfortable interacting with someone with schizophrenia or bipolar disorder, and $77 \%$ would not be comfortable going on a "date" with such a person (http:// www.mentalhealthamerica.net/go/surveys). Indeed, in our analysis of Americans' preferences for social distance from persons with schizophrenia between 1996 and 2006, we found no change in levels of rejection across either more intimate or less intimate interactional venues (Pescosolido et al. 2010). Similar patterns of the stability of stigma associated with schizophrenia have been found cross-nationally with regard to both social distance preferences and assessments of the unpredictability of persons with schizophrenia (e.g., Germany and New Zealand; Akroyd and Wyllie 2002; Angermeyer and Dietrich 2006, respectively). Further, data indicate that perceptions of the danger posed by persons with mental illnesses have significantly increased over the past 45 years (Phelan et al. 2000). Finally, media can have both negative and positive effects. For example, a study conducted in Germany documented a marked increase in social distance preferences from persons with schizophrenia after violent attacks on public figures (Angermeyer and Matschinger 1996).

\section{Policy Implications}

Patel and colleagues (2006) point out that there are at least two reasons to pursue the WHO paradox. On a basic level, data supporting or contradicting these ideas provide the scientific foundation for health needs and health policies. But more important, if sociocultural factors are in operation regarding how outcomes differ across national contexts, findings suggest a lever for interventions that target the community, the treatment system, and their interface. Indeed, past research has documented that culture holds the resources that individuals, families, and others in the community use to understand the experience of mental illness; categorize problems, prospects, and sources of care; and make decisions about their own, and often others', behavior (Keusch, Wilentz, and Kleinman 2006). In fact, Lefley (1990) contends that chronicity, itself, is a cultural artifact based at least in part on differing worldviews. 
It is not novel to suggest that studies that can directly address the WHO better prognosis hypothesis might be useful and that sociocultural characteristics, alongside clinical factors, should be included as a critical part of such investigations. Nor is it uncommon to recognize that such studies are unlikely to be funded or even useful in changing societies where over a third of the population lives on less than a dollar a day (Patel et al. 2006; Yang and Kleinman 2008). However, we disagree with much of the discussion surrounding the WHO paradox suggesting that researching the processes associated with recovery for persons with severe mental illness is either futile or unnecessary. As McGrath (2008, p. 247) points out, "we still struggle to understand the offset of schizophrenia," even as great strides have been made in studying its onset.

To be fair, WHO researchers have argued that the WHO paradox is "unlikely to be reducible to a single variable or to a small number if explanatory variables" (Jablensky 2009, p. 1459). To that end, we examined one aspect of the sociocultural environment - the larger national context of stigma - and found it to be wanting in providing support for unraveling the paradox. The idea that better outcomes in ISoS sites can be ascribed to more tolerant cultural environments can be removed from the list of mechanisms thought to be in operation. Overall, public stigma appears to be generally unrelated to a country's level of development, and where significant associations can be documented, public stigma tends to contrast with expectations under the WHO paradox. Assessing global needs and developing scientific foundations for improving treatment systems and community integration for persons with schizophrenia requires an understanding of the environmental factors, including the larger social context, that may hinder use or recovery, harm practitioner treatment efforts, and forestall political action (Prince et al. 2007). 


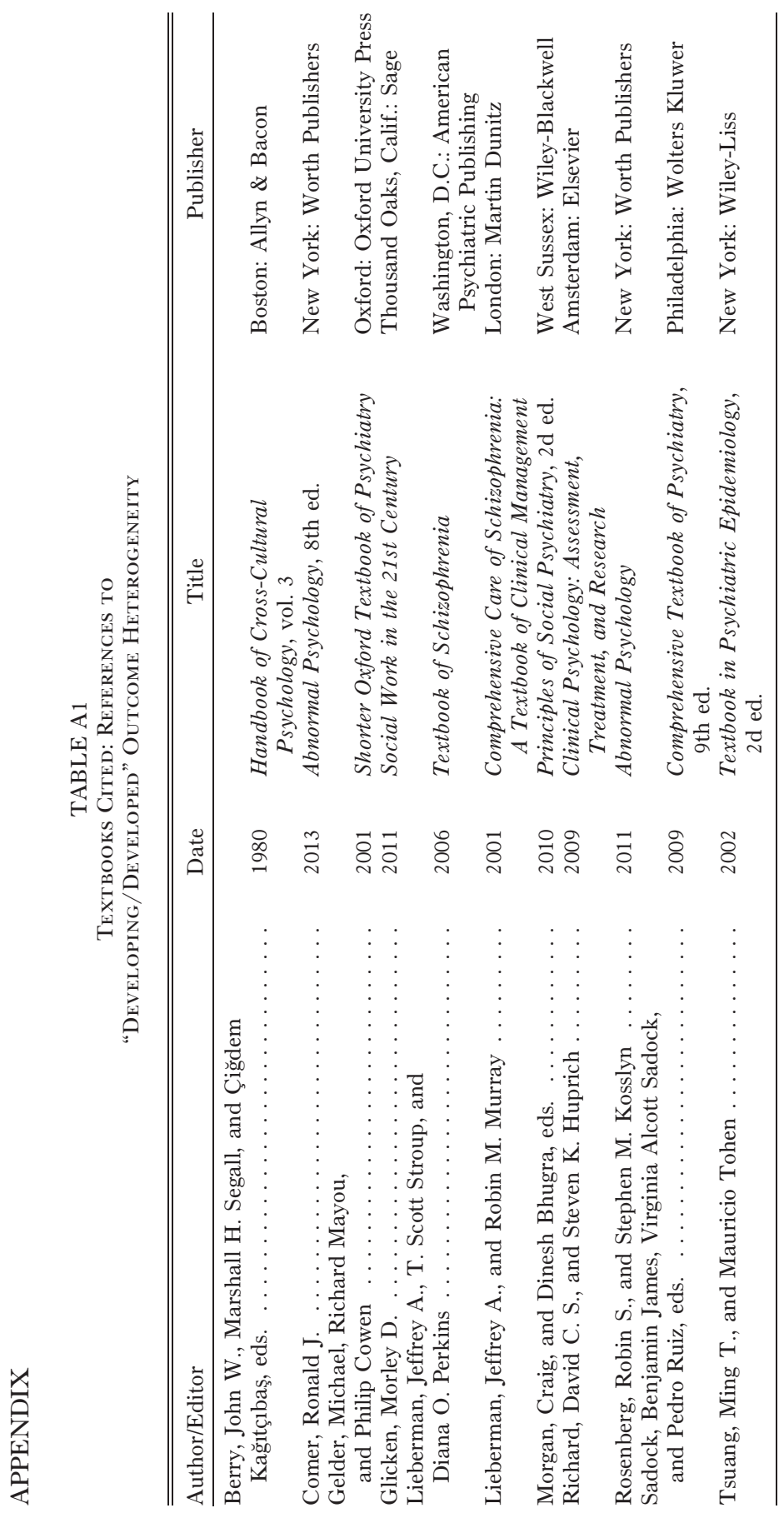

This content downloaded from 131.193.153.188 on April 18, 2016 08:07:04 AM All use subject to University of Chicago Press Terms and Conditions (http://www.journals.uchicago.edu/t-and-c). 


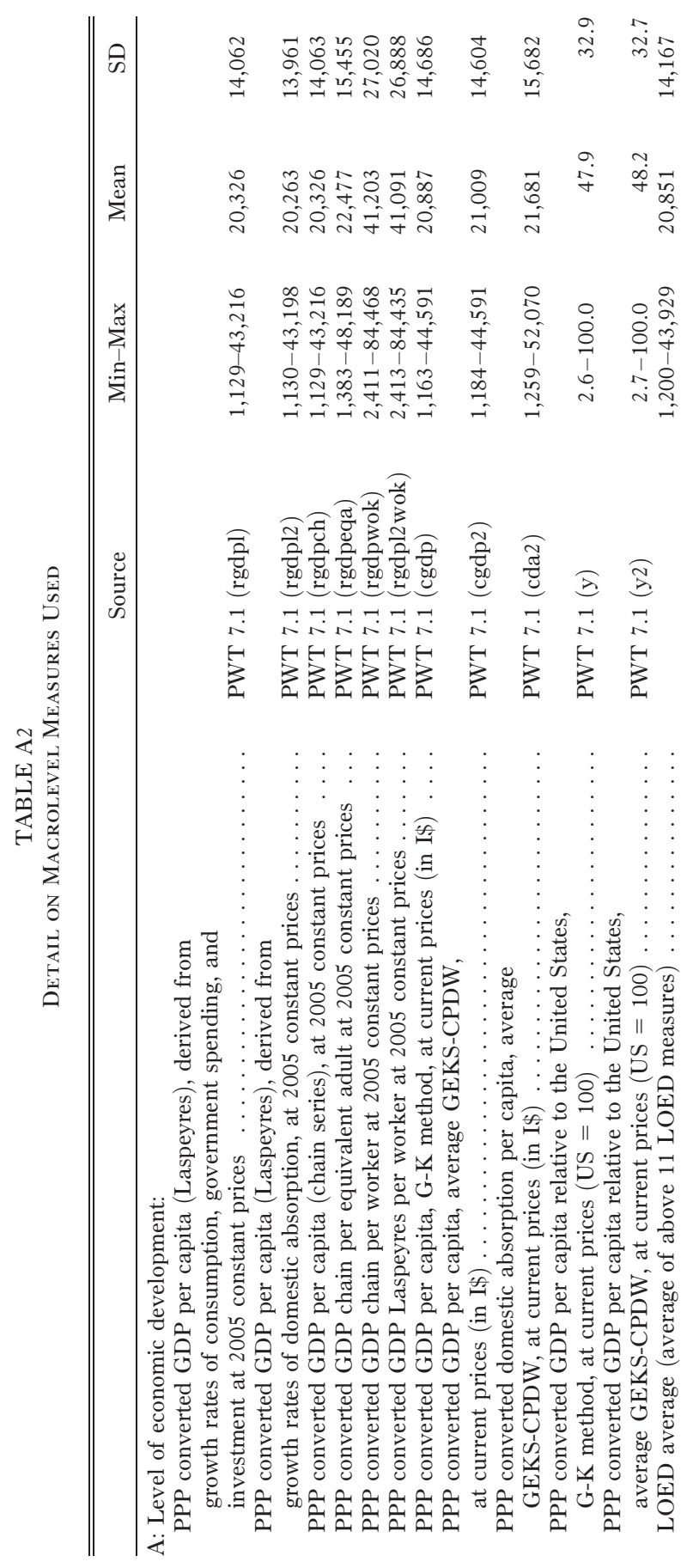




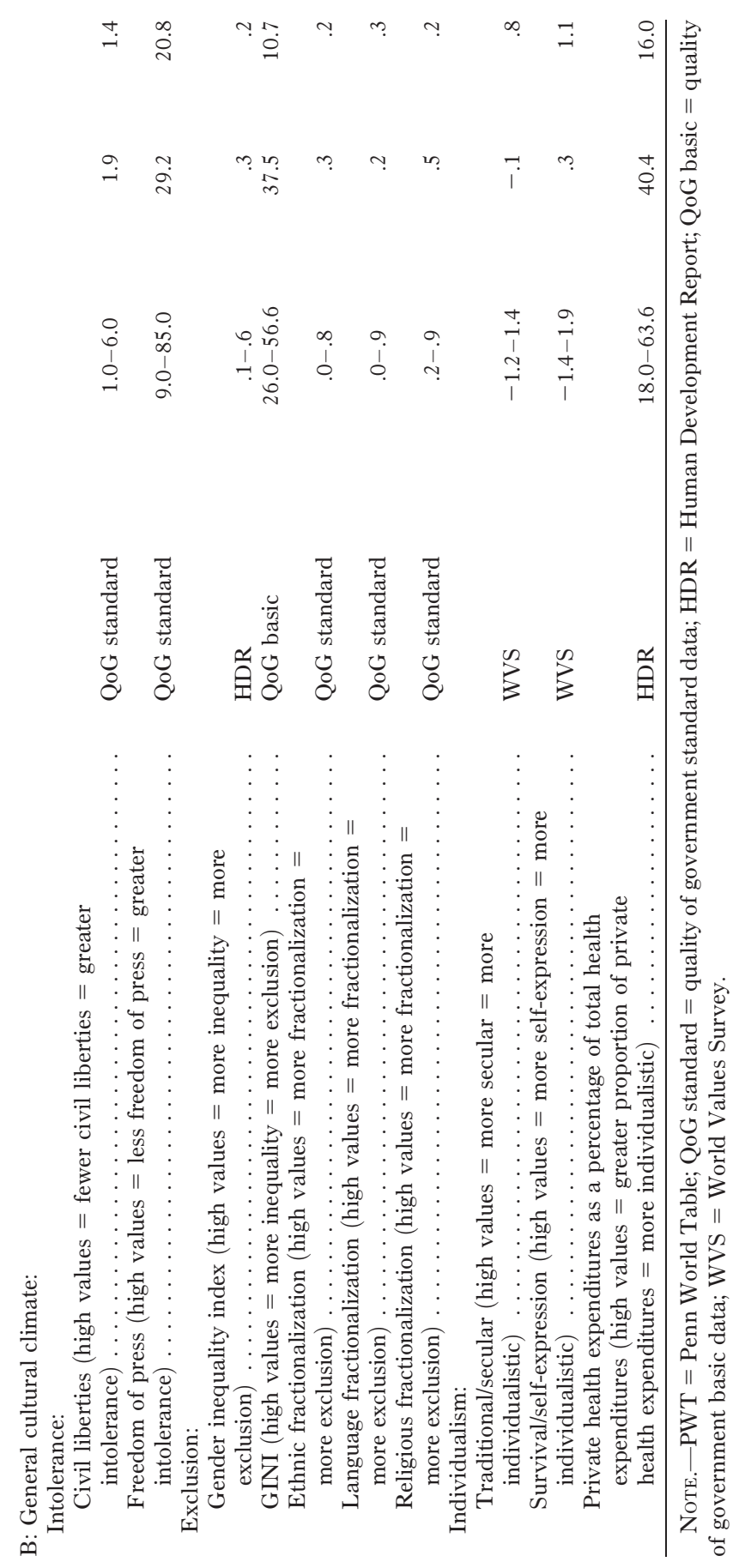




\section{American Journal of Sociology}

\section{REFERENCES}

Akroyd, S., and A. Wyllie. 2002. Impacts of a National Media Campaign to Counter Stigma and Discrimination Associated with Mental Illness: Survey 4. Auckland: Phoenix Research.

Angermeyer, Matthias C., and S. Dietrich. 2006. "Public Beliefs about and Attitudes towards People with Mental Illness: A Review of Population Studies." Acta Psvchiatrica Scandinavia 113 (3): 163-79.

Angermeyer, Matthias C., and Herbert Matschinger. 1996. "The Effect of Personal Experience with Mental Illness and Attitude toward Individuals Suffering from Mental Disorders." Social Psvchiatrv and Psvchiatric Epidemiologv 31:321-26.

Apiquian, Rogelio, Rosa-Elena Ulloa, Ana Fresan, and Humberto Nicolini. 2006. "The Impact of Duration of Untreated Psychosis (DUP) on Clinical Outcome in a 12-Month Follow-Up Study with Mexican Patients with First-Episode Psychosis.” Pp. 151-68 in Schizophrenic Psychology: New Research, edited by Douglas P. French. New York: Nova Science.

Beardsley, L., and P. Pedersen. 1980. "Health and Culture-Centered Intervention." Pp. 413-48 in Handbook of Cross-Cultural Psychology, edited by John W. Berry, Marshall H. Segall, and Çiğdem Kağıtçıbaş. Boston: Allyn \& Bacon.

Bellah, Robert N., Richard Madsen, William M. Sullivan, Ann Swidler, and Steven M. Tipton. 1985. Habits of the Heart: Individualism and Commitment in American Life. Berkeley and Los Angeles: University of California Press.

Benjamini, Yoav, and Yosef Hochberg. 1995. "Controlling the False Discovery Rate: A Practical and Powerful Approach to Multiple Testing." Journal of the Royal Statistical Society B 57 (1): 289-300.

Bresnahan, Michaeline, Paulo Menezes, Vijoy Varma, and Ezra Susser, eds. 2003. Geographical Variation in Incidence, Course, and Outcome of Schizophrenia: A Comparison of Developing and Developed Countries. Cambridge: Cambridge University Press.

Bromet, E. J., L. Jandorf, S. Fennig, J. Lavelle, et al. 1976. "The Suffolk County Mental Health Project: Demographic, Pre-morbid and Clinical Correlates of 6-Month Outcome." Psychological Medicine 26:953-62.

Brown, Phil. 1985. The Transfer of Care: Psychiatric Deinstitutionalization and Its Aftermath. London: Routledge \& Kegan Paul.

Burns, Jonathan K. 2009. "Dispelling a Myth: Developing World Poverty, Inequality, Violence and Social Fragmentation Are Not Good for Outcome in Schizophrenia." African Journal of Psychiatry 12:200-205.

Chatterjee, Sudipto, Neerja Chowdhary, Sulochana Pednekar, Alex Cohen, et al. 2008. "Integrating Evidence-Based Treatments for Common Mental Disorders in Routine Primary Care: Feasibility and Acceptability of the MANAS Intervention in Goa, India." World Psvchiatrv 7 (1): 39-46.

Cohen, Alex, Vikram Patel, R. Thara, and O. Gureje. 2008. "Questioning an Axiom: Prognosis for Schizophrenia in the Developing World?" Schizophrenia Bulletin 34 (2): 229-44.

Comer, Ronald J. 2013. Abnormal Psychology, 8th ed. New York: Worth

Corrigan, Patrick W. 2012. "Where Is the Evidence Supporting Public Service Announcements to Eliminate Mental Illness Stigma?" Psychiatric Services 63 (1): 79-82.

Couture, Shannon, and David L. Penn. 2003. "Interpersonal Contact and the Stigma of Mental Illness: A Review of the Literature." Journal of Mental Health 12:291-305.

Crisp, Arthur H., Michael Gelder, Susannah Rix, Howard Meltzer, and Olwen Rowlands. 2000. "Stigmatization of People with Mental Illness." British Journal of Psychiatry 177 (1): 4-7.

Deaton, Angus, and Alan Heston. 2010. "Understanding PPPs and PPP-Based National Accounts." Macroeconomics 2 (4): 1-35. 
Dillman, Don. 1978. "Implementing Mail Surveys." Pp. 160-99 in Mail and Telephone Surveys: The Total Design Method, edited by D. A. Dillman. New York: Wiley.

Edgerton, Robert B., and Alex Cohen. 1994. "Culture and Schizophrenia: The DOSMD Challenge." British Journal of Psychiatrv 164 (2): 222-31.

Evans-Lacko, Sara, E. Brohan, Ramin Mojtabai, and Graham Thornicroft. 2012. "Association between Public Views of Mental Illness and Self-Stigma among Individuals with Mental Illness in 14 European Countries." Psychological Medicine 42 (8): $1741-52$.

Feiner, Joel S., and Frederick J. Frese III, eds. 2009. "Recovery in Schizophrenia." Pp. 1582-93 in Kaplan and Sadock's Comprehensive Textbook of Psychiatry, 9th ed. Edited by B. J. Sadock, V. A. Sadock, and Pedro Ruiz. Philadelphia: Wolters Kluwer.

Fischer, Claude. 1982. To Dwell among Friends: Personal Networks in Town and City. Chicago: University of Chicago Press.

Fukuyama, Francis. 2001. "Social Capital, Civil Society and Development." Third World Ouarterly 22 (1): 7-20.

Gelder, Michael G., Richard Mayou, and Philip Cowen. 2001. Shorter Oxford Textbook of Psychiatry. Oxford: Oxford University Press.

Giddens, Anthony. 1972. "Four Myths in the History of Social Thought." Economy and Societv 1 (2): $357-85$.

. 1976. "Classical Social Theory and the Origins of Modern Society." American Journal of Sociologv 81 (4): 703-29.

Glicken, Morley D. 2011. Social Work in the 21st Century: An Introduction to Social Welfare, Social Issues, and the Profession. Thousand Oaks, Calif.: Sage.

Goffman, Erving. 1961. Asylums. Garden City, N.Y.: Anchor. 1963. Stigma: Notes on the Management of Spoiled Identity. Englewood Cliffs, N.J.: Prentice-Hall.

Greif, Avner. 1994. "Cultural Beliefs and the Organization of Society: A Historical and Theoretical Reflection on Collectivist and Individualist Societies." Journal of Political Economv 102 (5): 912-50.

Grob, Gerald N. 1991. From Asylum to Community: Mental Health Policy in Modern America. Princeton, N.J.: Princeton University Press.

Heston, Alan, Robert Summers, and Bettina Aten. 2012. "Penn World Table." Vers. 7.1. Center for International Comparisons of Production, Income, and Prices, University of Pennsylvania.

Hopper, Kim, ed. 2003. Interrogating the Meaning of 'Culture' in the WHO International Studies of Schizophrenia. New York: Cambridge University Press.

_ 2008. "Outcomes Elsewhere: Course of Psychosis in 'Other Cultures." Pp. 198216 in Society and Psychosis, edited by C. Morgan, K. McKenzie, and P. Fearon. Cambridge: Cambridge University Press.

Hopper, Kim, Glynn Harrison, Aleksandar Janca, and Norman Sartorius. 2007. Recovery from Schizophrenia: An International Perspective. Oxford: Oxford University Press.

Hopper, Kim, Glynn Harrison, and Joseph Wanderling. 2007. "An Overview of Course and Outcome in ISoS." Pp. 23-38 in Recovery from Schizophrenia: An International Perspective; A Report from the WHO Collaborative Project, the International Study of Schizophrenia, edited by K. Hopper, G. Harrison, A. Janca, and N. Sartorius. New York: Oxford University Press.

Hopper, Kim, and Joe Wanderling. 2000. "Revisiting the Developed versus Developing Distinction in Course and Outcome in Schizophrenia: Results from ISoS, the WHO Collaborative Follow-Up Project; International Study of Schizophrenia." Schizophrenia Bulletin 26 (4): 835-46.

Hopper, Kim, Joe Wanderling, and P. Narayanan. 2007. "To Have and to Hold: A Cross-Cultural Inquiry into Marital Prospects after Psychosis.” Global Public Health 2 (3): $257-80$. 


\section{American Journal of Sociology}

Inglehart, Ronald, and Wayne E. Baker. 2000. "Modernization, Globalization, and the Persistence of Tradition: Empirical Evidence from 65 Societies.” American Sociological Review 65:19-55.

Isaac, Mohan, Prabhat Chand, and Pratima Murthy. 2007. "Schizophrenia Outcome Measures in the Wider International Community." British Journal of Psychiatrv 191: S71-S77.

Jablensky, Assen, and Norman Sartorius. 2008. "What Did the WHO Studies Really Find?" Schizophrenia Bulletin 34 (2): 253-55.

Jablensky, Assen, Norman Sartorius, and J. E. Cooper. 1994. "Culture and Schizophrenia: Criticisms of WHO Studies Are Answered." British Journal of Psychiatry $165: 434-36$.

Jablensky, Assen, Norman Sartorius, G. Ernberg, M. Anker, et al. 1992. "Schizophrenia: Manifestations, Incidence and Course in Different Cultures; A World Health Organization Ten-Country Study.” Monograph suppl. Psvchological Medicine 20:1-97.

Jablensky, Assen V. 2009. "Course and Outcome of Schizophrenia and Their Prediction." In The New Oxford Textbook of Psychiatry, 2d ed. Edited by Michael G. Gelder, Nancy Andreasen, Juan Lopez-Ibor, and John Geddes. Oxford: Oxford University Press.

Kadri, Nadia, and Norman Sartorius. 2005. "The Global Fight against the Stigma of Schizophrenia." PLoS Medicine 2 (7): e136.

Kadushin, Charles. 1983. "Mental Health and the Interpersonal Environment: Reexamination of Some Effects of Social Structure on Mental Health." American Sociological Review 48:188-98.

Karagianis, Jamie, D. Novick, Jan Pecenak, Josep Maria Haro, et al. 2009. "WorldwideSchizophrenia Outpatient Health Outcomes (W-SOHO): Baseline Characteristics of Pan-Regional Observational Data from More than 17,000 Patients." International Journal of Clinical Practice 63 (11): 1578-88.

Keusch, Gerald T., Joan Wilentz, and Arthur Kleinman. 2006. "Stigma and Global Health: Developing a Research Agenda." Lancet 367 (9509): 525-27.

Kohn, Melvin L., and Robin M. Williams. 1956. "Situational Patterning in Intergroup Relations." American Sociological Review 21:164-74.

Kohn, Robert, Ronald M. Wintrob, and Renato D. Alarcon. 2009. "Transcultural Psychiatry." Pp. 734-53 in Kaplan and Sadock's Comprehensive Textbook of Psychiatry, 9th ed. Edited by B. J. Sadock, V. A. Sadock, and Pedro Ruiz. Philadelphia: Wolters Kluwer.

Krugman, Paul R. 1997. Pop Internationalism. Cambridge, Mass.: MIT Press.

Kulhara, Parmanand, and Subho Chakrabarti. 2001. "Culture and Schizophrenia and Other Psychotic Disorders." Psychiatric Clinics of North America 24 (3): 449-64.

Kulhara, Parmanand, Ruchita Shah, and Sandeep Grover. 2009. "Is the Course and Outcome of Schizophrenia Better in the 'Developing' World?" Asian Journal of Psychiatrv 2 (2): 55-62.

Lauronen, E., J. Miettunen, B. Veijola, M. Karhu, et al. 2007. "Outcome and Its Predictors in Schizophrenia within the Northern Finland 1966 Birth Cohort." European Psychiatry 22 (2): 129-36.

Laursen, Thomas Munk. 2011. "Life Expectancy among Persons with Schizophrenia or Bipolar Affective Disorder." Schizophrenia Research 131 (1): 101-4.

Lee, Sing, Margaret T. Y. Lee, Marcus Y. L. Chiu, and Arthur Kleinman. 2005. "Experience of Social Stigma by People with Schizophrenia in Hong Kong." British Journal of Psychiatry 186:153-57.

Leff, Julian 2001a. "Cultural InEuences on Schizophrenia." Pp. 243-55 in Comprehensive Care of Schizophrenia: A Textbook of Clinical Management, edited by Jeffrey A. Lieberman and Robin M. Murray. London: Martin Dunitz. 2001b. "Family Therapy in Psychiatry." In World Health Report, 2001. Geneva: World Health Organization. 
Leff, Julien, Norman Sartorius, Assen Jablensky, Alisa E. Korten, and G. Ernberg. 1992. "The International Pilot Study of Schizophrenia: Five-Year Follow-Up Findings." Psychological Medicine 22:131-45.

Lefley, Harriet P. 1990. "Culture and Chronic Mental Illness." Hospital and Community Psychiatry 41 (3): 277-86.

Lehmann, Erich L., and Joseph P. Romano. 2005. Testing Statistical Hypotheses. New York: Wiley.

Lin, Keh-Ming, and Arthur M. Kleinman. 1988. "Psychopathology and Clinical Course of Schizophrenia: A Cross-Cultural Perspective." Schizophrenia Bulletin 14 (4): 555-67.

Link, Bruce G., Howard Andrews, and Francis T. Cullen. 1992. "The Violent and Illegal Behavior of Mental Patients Reconsidered.” American Sociological Review 57:275-92.

Link, Bruce G., and Jo C. Phelan. 2001. "Conceptualizing Stigma." Annual Review of Sociology 27:363-85.

Loganathan, S., and S. R. Murthy. 2008. "Experiences of Stigma and Discrimination Endured by People Suffering from Schizophrenia." Indiana Journal of Psychology 50 (1): 39-46.

McGrath, John. 2008. "Dissecting the Heterogeneity of Schizophrenia Outcomes." Schizophrenia Bulletin 34 (2): 247-48.

Menon, M. S., and R. Shankar. 1993. "Family and Professionals Working Together in the Management of Schizophrenia." In Mental Health in India, edited by Purnima N. Mane and Katy Y. Gandevia. Delhi: Tata Institute of Social Sciences.

Meyer, John W., John Boli, George M. Thomas, and Francisco O. Ramirez. 1997. "World Society and the Nation-State." American Journal of Sociology 103 (1): 144-81.

Miller, Brian J., C. Bayard Paschall III, and Dale P. Svendsen. 2006. "Mortality and Medical Comorbidity among Patients with Serious Mental Illness." Psychiatric Services 57:1482-87.

Mojtabai, Ramin. 2010. "Mental Illness Stigma and Willingness to Seek Mental Health Care in the European Union." Social Psychiatry and Psychiatric Epidemiology 45: 705-12.

Ostrom, Elinor. 2009. "A General Framework for Analyzing Sustainability of SocialEcological Systems." Science 325:419-22.

Patel, Vikram, Alex Cohen, Thara Rangaswamy, and Oye Gureje. 2006. "Is the Outcome of Schizophrenia Really Better in Developing Countries?" Revista Brasileira de Psiquiatria 28 (2): 149-52.

Peralta, Victor, and Manuel J. Cuesta. 2011. "Neuromotor Abnormalities in NeurolepticNaive Psychotic Patients: Antecedents, Clinical Correlates, and Prediction of Treatment Response." Comprehensive Psychiatrv 52 (2): 139-45.

Perkins, Diana O., and Jeffrey A. Lieberman. 2006. "Natural History and Predictors of Clinical Course.” Pp. 289-301 in Textbook of Schizophrenia, edited by Jeffrey A. Lieberman, T. Scott Stroup, and Diana O. Perkins. Washington, D.C.: American Psychiatric.

Pescosolido, Bernice A. 2006. "Of Pride and Prejudice: The Role of Sociology and Social Networks in Integrating the Health Sciences." Journal of Health and Social Behavior 47 (September): 189-208.

. 2013. "The Public Stigma of Mental Illness: What Do We Think, What Do We Know, What Can We Prove?" Journal of Health and Social Behavior 54 (1): 1-21.

Pescosolido, Bernice A., Carol Brooks-Gardner, and Keri M. Lubell. 1998. "How People Get into Mental Health Services: Stories of Choice, Coercion and 'Muddling Through' from 'First-Timers." Social Science and Medicine 46 (2): 275-86.

Pescosolido, Bernice A., and Jack K. Martin. 2015. "The Stigma Complex." Annual Review of Sociology 41. doi:10.1146/annurev-soc-071312-145702.

Pescosolido, Bernice A., Jack K. Martin, Annie Lang, and Sigrun Olafsdottir. 2008. "Rethinking Theoretical Approaches to Stigma: A Framework Integrating Normative Influences on Stigma (FINIS)." Social Science and Medicine 67:431-40. 


\section{American Journal of Sociology}

Pescosolido, Bernice A., Jack K. Martin, Bruce Link, Saeko Kikuzawa, et al. 2000. Americans' Views of Mental Illness and Health at Century's End: Continuity and Change. Public report on the MacArthur Mental Health Module, 1996 General Social Survey. Bloomington: Indiana Consortium for Mental Health Services Research.

Pescosolido, Bernice A., Jack K. Martin, J. Scott Long, Tait Medina, et al. 2010. "'A Disease Like Any Other'? A Decade of Change in Public Reactions to Schizophrenia, Depression and Alcohol Dependence." American Journal of Psychiatry 167:1321-30.

Pescosolido, Bernice A., Tait R. Medina, Jack K. Martin, and J. Scott Long. 2013. "The 'Backbone' of Stigma: Identifying the Global Core of Public Prejudice Associated with Mental Illness." American Journal of Public Health 103 (5): 853-60.

Pescosolido, Bernice A., and Sigrun Olafsdottir. 2008. "The Logistics of Survey Implementation in a Comparative Study of Mental Illness: Issues and Resolutions in Translation across Cultural Boundaries." Presented at the International Conference on Survey Methods in Multinational, Multiregional, and Multicultural Contexts, Berlin.

Pescosolido, Bernice A., and Beth A. Rubin. 2000. "The Web of Group Affiliations Revisited: Social Life, Postmodernism, and Sociology." American Sociological Review 65 (February): 52-76.

Phelan, Jo C., Bruce G. Link, Ann Stueve, and Bernice A. Pescosolido. 2000. "Public Conceptions of Mental Illness in 1950 and 1996: What Is Mental Illness and Is It to Be Feared?" Journal of Health and Social Behavior 41 (2): 188-207.

Portes, Alejandro. 1976. "The Sociology of National Development." American Journal of Sociology 82:55-85.

Prince, Martin, Vikram Patel, Shekhar Saxena, Maria Maj, Joanna Maselko, Michael R. Phillips, and Atif Rahman. 2007. "No Health without Mental Health." Lancet 370 (9590): 859-77.

Ridgeway, Cecilia L. 2006. "Linking Social Structure and Interpersonal Behavior: A Theoretical Perspective on Cultural Schemas and Social Relations." Social Psychology Quarterly 69:5-16.

Rosenberg, Robin S., and Stephen M. Kosslyn. 2011. Abnormal Psychology. New York: Worth.

Rueschemeyer, Dietrich, Evelyne Huber Stephens, and John D. Stephens. 1992. Capitalist Development and Democracy. Chicago: University of Chicago Press.

Sartorius, Norman. 1997. "Fighting Schizophrenia and Its Stigma: A New World Psychiatric Association Educational Programme.” British Journal of Psychiatry 170 (4): 297.

Sartorius, Norman, Wolfgang Gulbinat, G. Harrison, E. Laska, and C. Siegel. 1996. "Long-Term Follow-Up of Schizophrenia in 16 Countries: A Description of the International Study of Schizophrenia Conducted by the World Health Organization." Social Psychiatry and Psychiatric Epidemiology 31 (5): 249-58.

Sartorius, Norman, Assen Jablensky, Alisa Korten, G. Ernberg, et al. 1986. "Early Manifestations and First-Contact Incidence of Schizophrenia in Different Cultures: A Preliminary Report on the Initial Evaluation Phase of the WHO Collaborative Study on Determinants of Outcome of Severe Mental Disorders." Psychological Medicine 16:909-28.

Sartorius, Norman, Assen Jablensky, and R. Shapiro. 1978. "Cross-Cultural Differences in the Short-Term Prognosis of Schizophrenic Psychoses." Schizophrenia Bulletin 4 (1): $102-13$.

Sartorius, Norman, and Hugh Schulze. 2005. Reducing the Stigma of Mental Illness: A Report from a Global Association. New York: Cambridge University Press.

Schomerus, Georg, C. Schwahn, Anita Holzinger, Patrick W. Corrigan, et al. 2012. "Evolution of Public Attitudes about Mental Illness: A Systematic Review and MetaAnalysis." Acta Psychiatrica Scandinavia 125 (6): 440-52. 
Shrivastava, Amresh, K., and Megan E. Johnston. 2010. "Cognitive Neurosciences: A New Paradigm in Management and Outcome of Schizophrenia." Indian Journal of Psychiatry 52:100-105.

Simmel, Georg. 1955. Conflict and the Web of Group Affiliations. New York: Free Press.

Star, Shirley A. 1955. "The Public's Idea about Mental Illness." Paper presented at the annual meeting of the National Association for Mental Health, Indianapolis, November 5 .

Stephens, John D., Evelyne Huber, and Leonard Ray. 1999. "The Welfare State in Hard Times." Pp. 164-93 in Continuity and Change in Contemporary Capitalism, edited by P. Lange, H. Kitchelt, G. Marks, and J. D. Stephens. Cambridge: Cambridge University Press.

Stroup, T. Scott, and Joseph P. Morrissey. 2001. "Systems of Care for Persons with Schizophrenia in Different Countries." Pp. 315-26 in Comprehensive Care of Schizophrenia: A Textbook of Clinical Management, edited by Jeffrey A. Lieberman and Robin M. Murray. London: Martin Dunitz.

Stuart, Heather, Julio Arboleda-Florez, and Norman Sartorius. 2012. Paradigms Lost: Fighting Stigma and the Lessons Learned. New York: Oxford University Press.

Swidler, Ann. 2001. Talk of Love: How Culture Matters. Chicago: University of Chicago Press.

Thara, Rangaswamy, and Tioupati Srinivasan. 2000. "How Stigmatizing Is Schizophrenia in India?" International Journal of Social Psychiatry 46:135-41.

Thirithalli, Jagadisha, and Sanjeev Jain. 2009. "Better Outcome of Schizophrenia in India: A Natural Selection against Severe Forms?" Schizophrenia Bulletin 35 (3): 655-57.

Waxler, Nancy, E. 1979. "Is Outcome for Schizophrenia Better in Nonindustrial Societies? The Case of Sri Lanka." Journal of Nervous and Mental Disease 167:144-58.

Whitaker, Robert. 2001. Mad in America. New York: Perseus.

Yang, Lawrence H., and Arthur Kleinman. 2008. "Face' and the Embodiment of Stigma in China: The Cases of Schizophrenia and AIDS." Social Science and Medicine 67:398-408. 